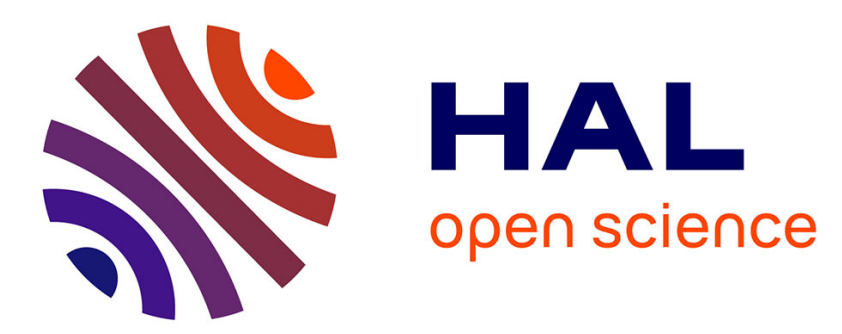

\title{
Simulation of Vertical Surfactant Distributions in Drying Latex Films
}

\author{
A. Gromer, F. Thalmann, P. Hébraud, Y. Holl
}

\section{To cite this version:}

A. Gromer, F. Thalmann, P. Hébraud, Y. Holl. Simulation of Vertical Surfactant Distributions in Drying Latex Films. Langmuir, 2017, 33 (2), pp.561-572. 10.1021/acs.langmuir.6b03913 . hal03586467

\section{HAL Id: hal-03586467 \\ https://hal.science/hal-03586467}

Submitted on 23 Feb 2022

HAL is a multi-disciplinary open access archive for the deposit and dissemination of scientific research documents, whether they are published or not. The documents may come from teaching and research institutions in France or abroad, or from public or private research centers.
L'archive ouverte pluridisciplinaire HAL, est destinée au dépôt et à la diffusion de documents scientifiques de niveau recherche, publiés ou non, émanant des établissements d'enseignement et de recherche français ou étrangers, des laboratoires publics ou privés. 


\title{
Simulation of Vertical Surfactant Distributions in Drying Latex Films
}

\author{
A. Gromer, ${ }^{1}$ F. Thalmann, ${ }^{1}$ P. Hébraud,${ }^{2}$ Y. Holl ${ }^{1, *}$ \\ 1. CNRS-ICS \& Université de Strasbourg \\ 23, rue du Loess BP 84047 \\ 67034 Strasbourg Cedex 2 \\ France \\ 2. IPCMS, 23 rue du Loess BP 43 \\ 67034 Strasbourg Cedex 2 \\ France \\ *yves.holl@unistra.fr
}

\begin{abstract}
:
Following our previous contribution (Langmuir 2015, 31, 10983) presenting a new simulation tool devoted to particle distributions in drying latex films, this article describes the prediction of surfactant concentration profiles in the vertical direction during the complete film formation process. The simulation is inspired by cellular automata and equations by Routh and coworkers. It includes effects that were not considered before: surfactant convection by water and surfactant desorption upon particle deformation. It is based on five parameters depending on the nature of the polymer / surfactant system and on the film formation conditions. Results show the importance of convection by water and the influence of the particular deformation mechanism on the final surfactant distribution. Excesses or depletions can be predicted either on the surface or on the substrate sides, in qualitative agreement with the numerous existing experimental studies. The complex interplay between parameters governing surfactant distributions makes the results unpredictable without the help of such a simulation tool. Therefore, it should be of interest to both industrial and academic scientists.
\end{abstract}




\section{INTRODUCTION}

Drying of colloids ${ }^{1}$ is the basic process by which many materials in form of thin films are prepared. ${ }^{2}$ One of the numerous theoretical and practical problems in colloid drying is the non trivial transport of matter during solvent evaporation. All species present in the colloid are prone to convective or diffusive transport, particles of all nature (organic polymer or inorganic) as well as hydrophilic, hydrophobic or amphiphilic molecules of all sizes. Convection and diffusion are in competition and the relative importance of the two phenomena is described by the dimensionless Peclet number. A Peclet number above one indicates that convection dominates with the possible consequence of species accumulation in some places in the drying system. If Peclet is below one, diffusion dominates, and the system remains more homogeneous. In the field of colloid drying, Peclet was defined $^{3}$ as the ratio $\frac{H \dot{E}}{D_{0}}, H$ being the deposit thickness, $\dot{E}$ the solvent evaporation rate in unit of length per unit of time (representing the speed of the downwards moving film / air interface) and $D_{0}$ the diffusion coefficient of the specie under consideration. This obviously means that different Peclet numbers are defined for different species.

Our article deals with the specific problem of surfactant distributions in drying of polymer colloids (latexes). This issue is of special interest for two reasons: (i) the surfactant distribution has a strong influence on the dry film properties ${ }^{4}$ (water uptake and permeability, surface and mechanical properties, tack and adhesion), and (ii) it is closely connected to the complex mechanisms of film formation from latexes and, to some extent, it sheds light on the subtleties of these mechanisms. ${ }^{4,5}$ This is why there is such a large amount of literature devoted to surfactant distribution in latex films. It is impossible to exhaustively quote all existing articles. A review of this topic was published in 1999 by Hellgren et al. ${ }^{6}$ A more recent review (2010) can be found in reference 4, chapter 6 . Surfactant distributions depend on all imaginable parameters: nature of the system (surfactant and latex polymer, inorganic pigments and fillers, substrate); physical characteristics of the latex (particle size and volume fraction, temperature, $\mathrm{pH}$, ionic strength, initial surfactant concentration) and of the deposit (size, shape, thickness); film formation (especially drying rate) and ageing conditions. The main features of the surfactant distributions published so far are the following. The surfactant can remain homogeneously distributed by staying adsorbed at the surface of the particles (or partly dissolved inside the latex polymer, which is possible with ethoxy based surfactants in acrylic latexes ${ }^{7,8}$ ). This is considered to be the most desirable case but it rarely happens. Very often, surfactant excesses at the film surface have been reported and discussed (see for example references 9 and 10). Actually, it has been recognized for a long time that almost all combinations of excess/depletion at both interfaces (film/air and film/substrate) were possible. ${ }^{7}$ It is also well documented that micron sized aggregates can form in the bulk of the film when the initial surfactant concentration exceeds the typical range of around $2 \mathrm{wt} \%$ (percent of dry content). ${ }^{11}$ On the other hand, polymerizable surfactants have attracted much interest in the nineties $^{12,13}$ because, being grafted at the surface of the particle, they are supposed to remain homogeneously distributed in the dry films. However, they complicate the latex synthesis, raising 
problems such as incomplete grafting, homopolymerization in the water phase, burying inside the particle.

A literature survey shows that the work on surfactant distribution is essentially experimental, modeling approaches are scarce. The main model is by Routh and coworkers. ${ }^{14,15}$ It considers the surfactant distribution in the vertical direction (the direction of the film thickness), in latexes of rigid particles. Lateral motions (parallel to the film surface) and edge effects are not taken into account. It describes the system evolution from the initial fluid dispersion state until particles come in contact over the whole film thickness (close packing), the interstices between particles still full of water. In the latex, the surfactant is partly in solution, partly adsorbed onto the latex particles, the amount adsorbed $\Gamma$ being related to the concentration in solution Cs through a Langmuir isotherm: $\Gamma=\frac{\Gamma_{\infty} C S}{A+C S}$, where $\Gamma_{\infty}$ is the maximum surface coverage onto the latex particles and A the solution concentration corresponding to $\Gamma=\Gamma_{\infty} / 2$. A is indicative of the initial slope of the isotherm, the smaller $\mathrm{A}$, the sharper the increase of the adsorbed amount. Surfactant transport through the drying film occurs in two ways: unadsorbed surfactants move through the serum while adsorbed surfactants are transported by latex particles (here transport means either diffusion or convection). The vertical distribution of surfactant depends on both the Langmuir isotherm parameters and the Peclet numbers of the particles $\left(\mathrm{Pe}_{\mathrm{p}}\right)$ and of the surfactants $\left(\mathrm{Pe}_{\mathrm{s}}\right)$. Depending on the values of these parameters, surfactant distributions showing enrichment or depletion at the interfaces could be predicted. Despite of the limitations of the model (rigid particles, incomplete drying), surfactant concentration measurements in poly(styrene-co-butyl acrylate) latexes using RBS (Rutherford Back Scattering) showed qualitative agreement with predictions. ${ }^{16}$

Another model, phenomenological in nature, was proposed by Arnold et al. ${ }^{11}$ to account for surfactant aggregate formation in the bulk of the dry film. In this model, the initial surfactant load is high enough to ensure particle surface saturation and micelle formation in water. After close packing of the particles, the air / water interface vertically sweeps over the particle array, downwards convecting the surfactant present in water and the one freed from the particle surface by desorption, in direction of the substrate. Consequently, the surfactant solution in water just below the air / water interface becomes more and more concentrated and viscous. When viscosity reaches a threshold value, transport is no longer possible, the drying front jumps over the obstacle, leaving a surfactant rich deposit (the future aggregate) and continues its way toward the substrate. The same process can start again to form another aggregate deeper in the film. This model draws attention upon two phenomena which might be important when thinking of surfactant distributions: the possibility of surfactant desorption when particles get close and deform and reduced mobility of water soluble species in systems where particles are already close packed and partially deformed.

This article presents a new theoretical approach of the problem of surfactant distributions in latex films based on a simulation, the first results of which concerned vertical distributions of particles during latex drying and were published recently. ${ }^{17}$ This simulation consists of dividing the drying system in space filing cubic cells exchanging matter (first water and particles, and here also surfactant) according to physical laws of increasing complexity. It is inspired by finite element simulations used in mechanics and by cellular automata. ${ }^{18}$ At each time step, the flux of matter in and out one cell depends on the states of its direct neighbors and obeys local rules. The evolution 
of the whole system is described by integrating the changing states of all cells over space and time. This approach allows an easier handling of the heterogeneous nature of drying colloids where moving drying fronts separating wetter and drier zones most usually appear. It has also the advantage of progressivity in increasing the complexity of treated cases in terms of dimensionality (1, 2 and 3D), number of constituents taken into account and nature of the considered physical laws (exchange of matter, but also heat and stress). Details about the software based on Object Oriented Programming using the Python free language are given in the next section.

The results presented here first reproduce the findings of Gundabala et al. ${ }^{14,15}$ and then refine them by taking into account convection of water (and surfactant contained in) associated to particle transport before treating the more complex case of the fate of the surfactant when particles undergo deformation. Our simulation integrates several assumptions (obviously not all of them) discussed in the literature into a description of the surfactant distribution through the complete drying and film formation process. 


\section{DESCRIPTION OF THE PROGRAM}

\subsection{Simulation Principles}

Here, we give a brief description of the main principles of our simulation. A more detailed description can be found in reference 17. Our approach is discrete and inspired by cellular automata. ${ }^{18}$ Space is divided into a set of fixed cells which can contain different compounds. The state of these compounds can change during the drying process. Depending on the state, different evolution rules apply, most of which describe exchanges of matter between neighboring cells. The latex contained in the cells can be in one of three main states: dispersion, wet gel or dry gel. In this context, gel means a state where particles, having started deformation or not, are in contact. In the wet gel, interstices between particles are full of water, in the dry gel they consist of air. When in the dispersion state, latex particle exchanges occur by diffusion and are calculated using a concentration dependent diffusion coefficient. ${ }^{17,}{ }^{19}$ In the gel states, exchanges of latex particles by convection are calculated based on the stress-strain equation governing the compacting particle network. ${ }^{17}$ In the vertical drying automaton, edge effects are neglected, hence, the system is onedimensional. As water evaporates, the air/water interface moves downward across the cells. The whole system goes through three drying stages (see Figure 1). In the first drying stage (or concentration stage), if evaporation is fast in comparison to particle diffusion, the latex can be both in the dispersion state (at the bottom of the film) and in the wet gel state (at the top). In the second drying stage, the latex is in the wet gel state in all cells and the whole particle network deforms under the capillary stress exerted at the film surface. In the third drying stage, the maximum capillary stress is reached and the particle network cannot deform any further, therefore a drying front moves downward through the film.

1)

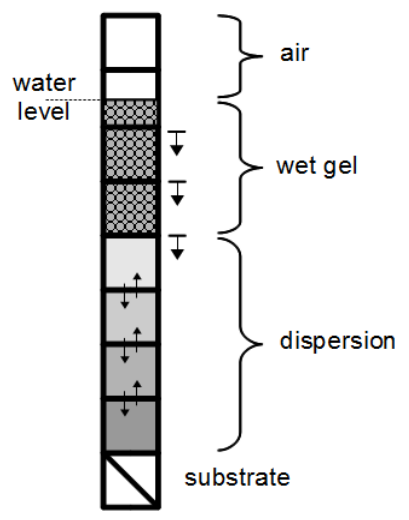

2)

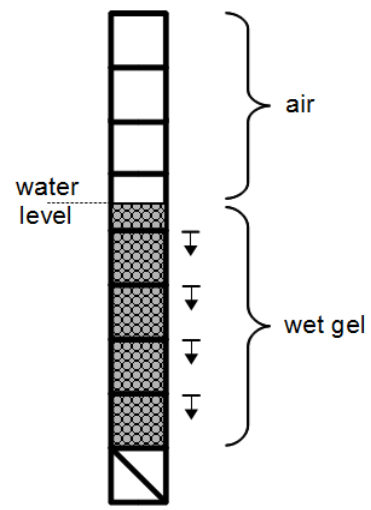

3)

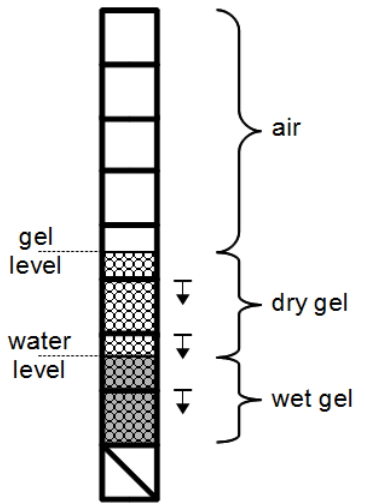

Figure 1. General Principles of the vertical drying simulation with three drying stages.

The Routh and Russel model ${ }^{3}$ is used to determine the stress and strain rates of the compacting particle network during the three drying stages. Here, the parameter $\bar{\lambda}$ (the evaporation rate divided by the compaction rate) is particularly important because it determines which deformation mechanism dominates (either wet sintering, capillary deformation, receding water 
front or dry sintering). The state variables and evolution rules related to the latex particles and the global drying mechanism were described in our previous paper and its Supporting Information. ${ }^{17}$ In the following section, the new variables and rules associated with the inclusion of surfactants into our program are described.

\subsection{New variables and rules added to the program}

The new variables associated with surfactants are gathered in Table 1. L designates the cell size and $1_{\text {water }}$ the water level in the cell.

\begin{tabular}{|c|l|}
\hline $\begin{array}{c}\text { CellSpace } \\
\text { (global) }\end{array}$ & $\begin{array}{l}\Gamma_{\infty}: \text { maximum surface adsorption onto the latex particles } \\
A: \text { solution concentration corresponding to half the maximum adsorption }\end{array}$ \\
\hline $\begin{array}{c}\text { Latex } \\
\text { (local) }\end{array}$ & $r_{s v}:$ surface / volume ratio of the latex particles \\
\hline $\begin{array}{c}\text { Surfactant } \\
\text { (local) }\end{array}$ & $\begin{array}{l}\text { Cs: surfactant concentration in serum } \\
\text { Csapp: apparent surfactant concentration in serum } \\
\text { Ds: surfactant diffusion coefficient } \\
\Gamma: \text { surface concentration of adsorbed surfactants } \\
S_{\text {sol }}: \text { concentration of non-adsorbed surfactants in the cell } \\
\left(\mathrm{S}_{\text {sol }}=c_{S}\left(\frac{l_{\text {water }}}{L}-\phi\right)\right) \\
S_{a d s}: \text { concentration of adsorbed surfactants in the cell }\left(\mathrm{S}_{\mathrm{ads}}=\Gamma \cdot r_{s v}\right) \\
S_{\text {tot }}: \text { total concentration of surfactants in the cell }\left(\mathrm{S}_{\mathrm{tot}}=\mathrm{S}_{\mathrm{sol}}+\mathrm{S}_{\mathrm{ads}}\right)\end{array}$ \\
\hline
\end{tabular}

Table 1. New variables added to the program.

The following evolution rules describe how these variables are updated in each cell and at every time step dt.

\section{Apparent concentration.}

The role of the apparent concentration $\mathrm{C}_{\text {Sapp }}$ is to allow us to calculate exchanges of surfactants between two cells in the case where an interface is going through one of them. When the latex is in the "dispersion" state, $\mathrm{C}_{\text {Sapp }}$ is calculated based on the water level $1_{\text {water }}$ in the cell and the mirror image principle (a similar argument was used in our previous paper to calculate the latex apparent volume fraction). Two cases can be distinguished: if $1_{\text {water }} \geq \mathrm{L} / 2$, then $\mathrm{C}_{\text {Sapp }}=\mathrm{Cs}$; otherwise, $\mathrm{C}_{\text {Sapp }}$ $=2 \mathrm{Cs}\left(21_{\text {water }} / \mathrm{L}\right)+\mathrm{Cs}^{\prime}\left(\mathrm{L}-21_{\text {water }}\right) / \mathrm{L}$, where Cs' is the surfactant concentration in the lower neighboring cell.

\section{Exchanges by diffusion in solution.}

For each neighboring cell (designated by an apostrophe) whose latex is either in the dispersion state or the wet gel state, an exchange of surfactants is calculated using:

$d S_{\text {sol }}=\frac{D s\left(1-\phi_{a p p}+1-\phi_{a p p}^{\prime}\right)}{2}\left(C s_{a p p}^{\prime}-C s_{a p p}\right) \frac{d t}{L^{2}}$, where $\phi_{a p p}$ is the latex apparent volume fraction. ${ }^{17}$ 


\section{Exchanges by diffusion of latex particles.}

For each neighboring cell whose latex is in the dispersion state, an exchange of adsorbed surfactants is calculated using:

$d S_{a d s}=\frac{3}{2 R_{0}}\left(D_{p}\left(\phi_{a p p}\right) \Gamma+D_{p}\left(\phi_{a p p}{ }^{\prime}\right) \Gamma^{\prime}\right)\left(\phi_{a p p}{ }^{\prime}-\phi_{a p p}\right) \frac{d t}{L^{2}}$, where $D_{p}$ is the latex particle diffusion coefficient and $R_{0}$ the particle radius.

When the latex is in the wet gel state, the exchange of adsorbed surfactants is calculated using:

$d S_{a d s}=\Gamma r_{s v}\left(\phi_{a p p}\right) \frac{v_{g e l} d t}{l_{\text {water }}}$, where $v_{g e l}$ is the latex particle velocity.

\section{Exchanges by convection of water.}

For every exchange of latex particles between the cells, an equal volume of water is exchanged in the opposite direction (further discussed in next section and in Supporting Information). When the latex is in the dispersion state, the exchange of surfactant convected by water is given by:

$d S_{a d s}=\frac{-\left(D_{p}\left(\phi_{a p p}\right) C s+D_{p}\left(\phi_{a p p}{ }^{\prime}\right) C s^{\prime}\right)}{2}\left(\phi_{a p p}{ }^{\prime}-\phi_{a p p}\right) \frac{d t}{L^{2}}$

When the latex is in the gel state, the exchange of surfactants is given by:

$d S_{a d s}=C s^{\prime} \phi_{a p p} \frac{v_{g e l} d t}{L}$

\section{Langmuir adsorption onto latex particles.}

The surfactant concentration in the serum $C s$ and surface concentration of adsorbed surfactant $\Gamma$ are re-calculated at every time step from the total surfactant concentration $\mathrm{S}_{\text {tot }}=\mathrm{S}_{\text {sol }}+\mathrm{S}_{\text {ads }}$ using the Langmuir isotherm $\left(\Gamma=\frac{\Gamma_{\infty} C s}{A+C s}\right)$. This amounts to solving the following quadratic equation in $C s$ using the discriminant method:

$\left(\frac{l_{\text {water }}}{L}-\phi_{a p p}\right) C s^{2}+\left(\left(\frac{l_{\text {water }}}{L}-\phi_{a p p}\right) A+\Gamma_{\infty} r_{s v}-S_{t o t}\right) C s-A S_{\text {tot }}=0$

Subsequently, $\Gamma$ is calculated using the Langmuir isotherm equation, and $\mathrm{S}_{\text {ads }}$ and $\mathrm{S}_{\text {sol }}$ are recalculated using their definitions.

\section{Surface / volume ratio of latex particles.}

When in the "dispersion" state, the surface / volume ratio of latex particles is calculated using: $r_{s v}=$ $3 \Phi / R o$. When in the "gel" state, we assume the latex particles to form a random close packed arrangement. Furthermore, we assume the particles in the network deform as troncated spheres. Since the surface / volume ratio of such a system is not available, we constructed a $5^{\text {th }}$ order polynomial function $r_{s v}(\Phi)$ so that its curve was intermediate between the surface / volume ratios of cubic and face centered cubic structures (the latter were calculated using simple geometric considerations). ${ }^{20,21}$ It was checked that the precise shape of the function between these two extremes had very little effect on the results.

To conclude this paragraph and to help clarify what our simulation does, we discuss it from the alternate point of view of partial differential equations. When the latex is in the dispersion state in the whole film, the application of the evolution rules described above is equivalent to solving 
the following conservation equation for the surfactant (in parallel with another relevant conservation equation for latex particles) with appropriate boundary conditions (i.e., no flux at the substrate and at the film surface):

$$
\frac{\partial}{\partial t}\left(C_{S}(1-\phi)+\Gamma r_{s v}(\phi)\right)=\frac{\partial}{\partial y}\left(D_{s}(1-\phi) \frac{\partial C_{S}}{\partial y}\right)+\frac{\partial}{\partial y}\left(\Gamma \frac{3}{R_{0}} D_{p}(\phi) \frac{\partial \phi}{\partial y}\right)-\frac{\partial}{\partial y}\left(C_{S} D_{p}(\phi) \frac{\partial \phi}{\partial y}\right)
$$

The first term on the right hand side of the equation describes the transport of surfactant molecules in solution by diffusion while the second term describes the transport of surfactants adsorbed onto diffusing latex particles. The same two terms appear in the equation solved by Gundabala et al. ${ }^{14}$ The difference is that, in our simulation, when the latex is in the gel state at a

particular position, the second term is replaced by a convection term: $-\frac{\partial}{\partial y}\left(v_{p} \Gamma r_{s v}(\phi)\right)$, where $v_{p}$ is the latex particle velocity which depends on the evaporation rate and the deformation rate (see previous article $\left.{ }^{17}\right)$.

Finally, the third term on the right hand side of the equation accounts for surfactant convection in water and has not been taken into account previously. A derivation of the corresponding transport equations by means of a variational principle is presented in Supporting Information. When the latex is in the gel state, this term is replaced by: $+\frac{\partial}{\partial y}\left(C_{s} v_{p} \phi\right)$. Note the difference in sign of this term which reflects the fact that the surfactants in water go in the opposite direction to the latex particles.

\subsection{Langmuir isotherm and choice of parameters}

For the adsorption of surfactants on polymer particles, the choice of a Langmuir isotherm ${ }^{22}$ may be questioned. Two fundamental requirements for applicability, absence of interactions between adsorbed species (problematic especially for ionic surfactants) and homogeneity of the solid surface (generally not the case for copolymers), seem not fulfilled. However, there are examples in the literature where experimental data are nicely fitted by a Langmuir equation. ${ }^{23}$ In some cases, a fitting parameter has to be used (an exponent on the solution concentration). ${ }^{24}$ Arnold et al. ${ }^{25}$ published isotherms, obtained through conductimetry measurements, showing an almost linear increase at low solution concentration followed by a horizontal plateau. A singular point between the two regimes corresponded to the CMC of the surfactant in the solution. The worst case, i.e. an isotherm shape very different from a Langmuir isotherm, is when a first plateau is followed by a sharp increase. ${ }^{26}$ It is difficult to find a rational for these differences. Nevertheless, except in the latest cited case ${ }^{26}$ it globally appears that the Langmuir isotherm is not too bad an approximation of the experimental adsorption results and is probably the best choice for a simulation study.

Among the various possible polymer / surfactant systems, two extreme situations can be distinguished: (i) a rather hydrophobic surfactant (bearing a long hydrophobic tail and a small, not or weakly charged hydrophilic head) strongly adsorbing on a rather hydrophobic polymer (polystyrene for example), and (ii), the opposite, a hydrophilic surfactant weakly adsorbing on a less hydrophobic polymer, like poly(vinyl acetate). In case (i), the Critical Micellar Concentration (CMC) of the surfactant will be low, most of it will adsorb at the polymer / water interface, 
repulsions in the adsorbed layer will be minimal, resulting in high adsorbed amounts. In terms of Langmuir parameters, A will be low, and $\Gamma_{\infty}$ high. In case (ii), the CMC is high, the surfactant has more affinity with the water phase, the adsorbed layer is not dense because of the repelling heads, A will be high and $\Gamma_{\infty}$ low. In our simulation, in order not to handle too many and somewhat arbitrarily varying parameters, we treated these two extreme cases and one intermediate. In the calculations, the real Langmuir parameters are scaled with respect to the initial bulk surfactant concentration $\left(C s_{0}\right): \overline{\mathrm{A}}=\mathrm{A} / C s_{0}$ and $\mathrm{k}=3 \Gamma_{\infty} / \mathrm{R} C s_{0}, \mathrm{R}$ being the particle radius. Considering a typical value for $C s_{0}$ (let us take $2 \mathrm{wt} \%$ with respect to the polymer), this leads to $\mathrm{k}=10$ and $\overline{\mathrm{A}}=0.1$ for case (i) (hydrophobic surfactant); $k=2$ and $\overline{\mathrm{A}}=10$ for case (ii) (hydrophilic polymer); $k=4$ and $\overline{\mathrm{A}}$ $=1$ for the intermediate case.

Recalling the definition of the Peclet number: $\mathrm{P}_{\mathrm{e}}=\frac{H \dot{E}}{D_{0}}$, (with $H$ the deposit thickness, $\dot{E}$ the solvent evaporation rate and $D_{0}$ the diffusion coefficient) and taking $D_{0}$ as the Stokes Einstein coefficient: $D_{0}=\frac{k T}{6 \pi \mu R}(k:$ Boltzmann constant; $T:$ temperature, $\mu$ : solvent viscosity; $R$ : radius of the specie under consideration), one sees that the Peclet numbers of the particle $\left(\mathrm{P}_{\mathrm{eP}}\right)$ and of the surfactant $\left(\mathrm{P}_{\mathrm{es}}\right)$ are in the ratio of the sizes of the two objects. Therefore, it is unrealistic to consider too close values for these two parameters. In this study (except otherwise stated), a ratio of 100 was respected. The values which were privileged are $\mathrm{P}_{\mathrm{eP}}=50$ and $\mathrm{P}_{\mathrm{eS}}=0.5$. This corresponds to standard (rather fast) drying conditions and to a small, typical surfactant like Sodium Dodecyl Sulfate (SDS).

Deformation of the particles during (or after) drying was characterized by the dimensionless parameter $\bar{\lambda}$ (evaporation rate divided by deformation rate) by Routh and Russel. ${ }^{3}$ The various possible deformation mechanisms were associated to different values of $\bar{\lambda}$ in a global deformation map (see also reference 4 for more explanations on this approach). In our study, we successively treated the cases of capillary deformation $(\bar{\lambda}=25)$ (starting with this case because it is often considered as the most standard one); wet sintering $(\bar{\lambda}=0.5)$ (particle deforming in presence of water); and receding water front $(\bar{\lambda}=200)$ (slow capillary deformation, in such a way that the air / water interface penetrates into the film before total deformation and sweeps the particle array from top to bottom).

The last two parameters to consider are time and position in the film thickness. Time is scaled with respect to the end of the first drying step. The first drying step is defined here as the state of the system where all particles are in contact (in a random array) throughout the film thickness, the interstices between particles remaining full of water. The initial particle volume fraction is 0.4. Position is scaled with respect to the film thickness at the time the simulation is started (not necessarily at $\mathrm{t}=0$ ). The origin is at the bottom of the film (substrate side). 


\section{RESULTS AND DISCUSSION}

\subsection{First Drying Step}

In this paragraph, everything pertains to the first drying step. Using the same set of assumptions as Gundabala et al. ${ }^{14,15}$ (adsorption of the surfactant following a Langmuir isotherm, rigid particles moving in an immobile solvent, end of the process at the end of the first drying step), we ran our simulation in order to check whether it was able to reproduce the results of the model. Figure S1 in Supporting Information shows that the simulation almost perfectly fits the profiles calculated by Gundabala et al. It has to be noted that Gundabala et al. did not show surfactant distributions right at the end of the first drying step but slightly before (at $\mathrm{t} \sim 0.95$ if time is normalized by the time needed to reach close packing), presumably because of numerical instabilities (the diffusion coefficient diverges when the volume fraction reaches close packing). Our simulation does not suffer from this limitation for the reason exposed in reference 17.

The effects of the four parameters (Langmuir and Peclet) are abundantly commented in Gundabala et al.'s articles. ${ }^{14,15}$ Let us just briefly present and interpret the results obtained using our choice of parameters. Figure $2 \mathrm{a}$ shows surfactant distributions at the end of the first drying step $(t=1)$ for the three typical polymer / surfactant systems defined above: (i) hydrophobic surfactant $(\mathrm{k}=10 ; \overline{\mathrm{A}}$ $=0.1)$; (ii) hydrophilic surfactant $(\mathrm{k}=2 ; \overline{\mathrm{A}}=10)$; (iii) intermediate $(\mathrm{k}=4 ; \overline{\mathrm{A}}=1)$. Peclet numbers are as previously defined $\left(\mathrm{P}_{\mathrm{eP}}=50 ; \mathrm{P}_{\mathrm{eS}}=0.5\right)$. Surfactant concentrations are expressed in percent of excess or depletion compared to the hypothetical situation where the surfactant was distributed homogeneously throughout the film thickness. ${ }^{14}$ One can observe that in case (i), the distribution is almost uniform; whereas, for case (ii), the surface is at the nominal concentration but the substrate side is enriched while the inside of the film is depleted. As expected, case (iii) is intermediate. Figure $2 \mathrm{~b}$ showing the evolution of the surfactant distribution as drying proceeds towards close packing ( $t=0.85$ to 1$)$ for the intermediate case defined above ( $k=4$ and $\bar{A}=1)$, helps understanding the curve shapes in Fig. 2a. Upon drying, the surfactant solution in water concentrates, and, consequently, more surfactant is adsorbed at the polymer surface, in accordance with the specific isotherm associated to the concerned polymer / surfactant system. Adsorption might possibly stop if surface saturation is reached. If $\mathrm{P}_{\mathrm{eP}}$ is high (our case), a heterogeneity appears in the film: a top layer where particles are densely packed (at a volume fraction of 0.64) separated from a bottom, fluid zone by a more or less diffuse interface (often called the particle front). The top, dense layer progressively increases in thickness and the particle front moves downwards, toward the substrate. This is clearly seen in Fig. $2 \mathrm{~b}$ as the step in the distribution moving to the left. In the wet gel (right side of the step), the surfactant concentration is higher than in the fluid (left side). In the case considered in Fig. $2 b$, the partition of the surfactant between the surface of the particles and the solution is such that the gel, containing more particles and less solution, has more surfactant than the fluid, containing less particles and more solution. The fluid on the substrate side progressively concentrates by drying (here, just by reducing its volume). Correlatively, the concentration excess in the gel decreases because the total amount of surfactant in the film is obviously constant. The advancing particle front gives rise to a concentration increase due to the supply of the surfactant adsorbed at the surface of the particles in the gel (see for instance the concentration increase at $\mathrm{y} / \mathrm{H}=1.5$ between times 0.85 and 0.9 ). When the gel reaches the substrate, 
it provides surfactant adsorbed at the particle surfaces to an enriched solution, leading to an excess on the substrate side.

Looking back at Fig. 2a, one sees that this effect is stronger when the initial concentration in water is higher (case (ii)) and almost inexistent if the surfactant partition is in favor of the particle surfaces (case (i)). Figure 2 will serve as a comparison point to discuss the refinement of the model presented in the next paragraph. 

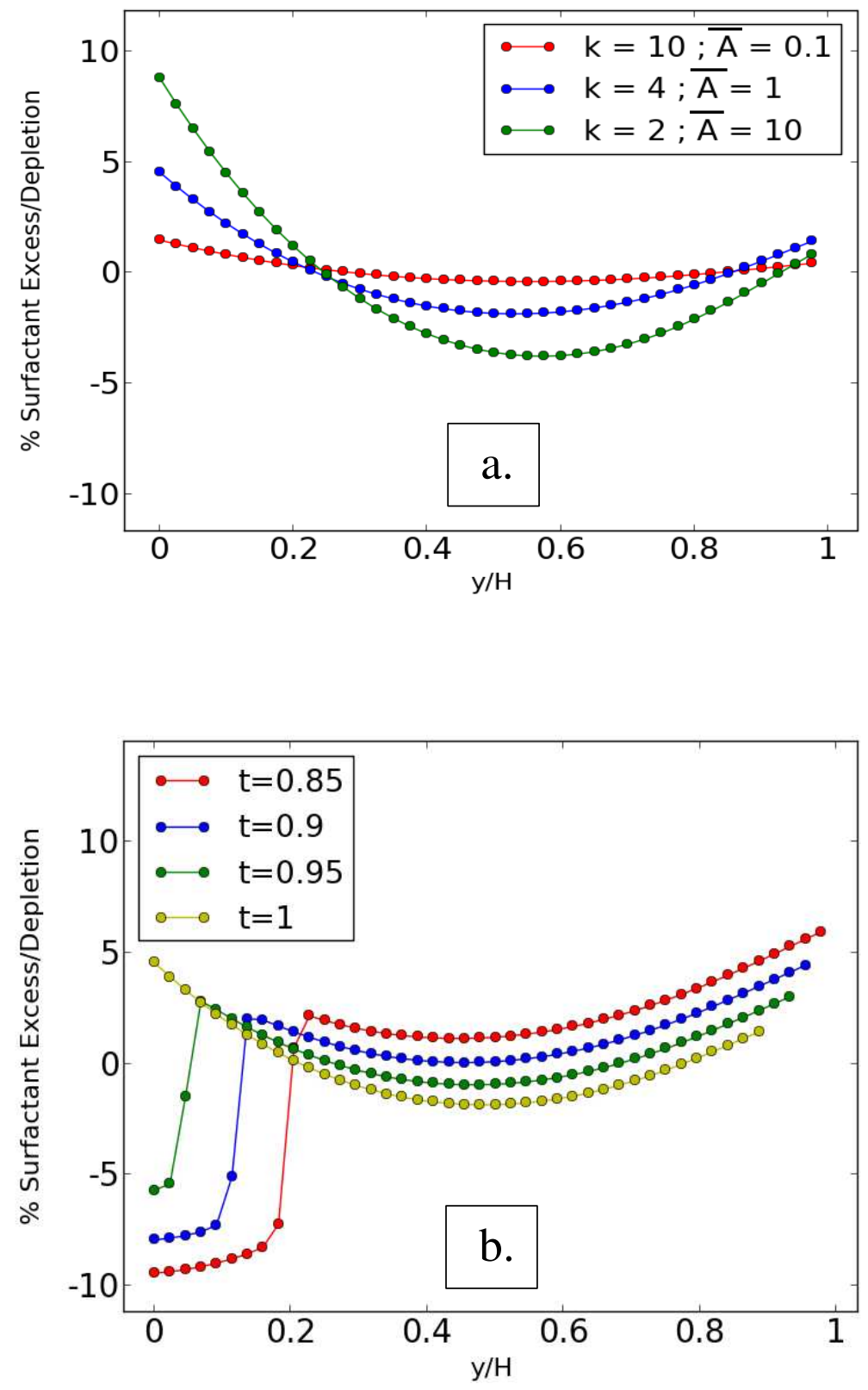

Figure 2. Percent surfactant excess/depletion as a function of scaled distance from the substrate in the vertical direction. Air / film surface on the right, substrate at zero, on the left. $\mathrm{P}_{\mathrm{eP}}=50 ; \mathrm{P}_{\mathrm{eS}}=0.5 ; \mathrm{t}=1$ at the end of the first drying step.

a. Surfactant distributions at $\mathrm{t}=1$ for different polymer/surfactant systems.

b. Evolution of the surfactant distribution at different times before close packing for an intermediate polymer/surfactant system: $\mathrm{k}=4 ; \overline{\mathrm{A}}=1$. 


\section{Convection of the Surfactant by Water During Drying}

The transport equations introduced and solved by Gundabala et al. ${ }^{14,15}$ correspond to a situation where latex particles move relatively to a stationary solvent. An improvement of the approach is proposed here, based on the simple idea that to every latex particle exchange between cells corresponds a flow of an equivalent volume of water (actually water plus surfactant) in the opposite direction. This flow has the effect of convecting surfactant in direction of the film surface because, in the conditions set by the parameters chosen in this work, the main motion of the particles is toward the substrate. A derivation of both Gundabala et al. transport equations, and our improved treatment of the surfactant convection consecutive to latex particle transport during the concentration stage is provided as Supplementary Information. Both approaches neglect the influence of surfactant concentration gradients on latex particle transport.

Figure 3 is equivalent to Fig. 2 but shows the considerable effect of convection by water. As could be expected, the upward transport of surfactant in water gives rise to an excess at the surface and a depletion at the substrate. The effect is even more marked that there is more surfactant in the water phase. When most of the surfactant is on the particle surface, the distribution remains almost uniform, like in the previous case (Fig. 2a). Figure 3b shows the discontinuity associated to the particle front. However, on the steps, the concentration relatively sharply increases toward the film surface. Interestingly, opposite to the previous situation (Fig. 2b), at intermediate times on the substrate side, the concentration progressively decreases meaning that the effect of convection overcomes that of drying. Nevertheless, the supply of surfactant by the wet gel as it touches the substrate make the concentration jump up again.

Surfactant excesses at the film surface have been reported a long time ago. ${ }^{27,28}$ Soon appeared the explanation that surfactant was carried to the surface by convection in water. ${ }^{7}{ }^{29}$ However, at that time, it was wrongly thought that drying could simply generate a neat vertical flux of water toward the surface, which is actually not possible because there is no water "reservoir" on the substrate side. The point remained unclear for a while. For instance, Tzitzinou et al., ${ }^{9}$ discussing surfactant excess at the film surface, spoke of migration without further precision ("...surfactant will migrate to the air/water interface..."). Later on, Mallégol et al. ${ }^{10}$ invoked the curved aqueous menisci on top of small capillaries formed by partially deformed particles on top of the film to draw water (plus surfactant) upward ("...just as water is drawn up a capillary tube against gravity."). To our knowledge, in the context of surfactant transport, it is the first time that the simple mechanism described above of a flux of water necessarily associated to a flux of particles in the opposite direction is proposed. In the remainder of the article, it will be systematically integrated in the phenomena taken into account in the simulation.

Before ending this paragraph, it has to be mentioned that, as already shown by Gundabala et

al. ${ }^{14,15}$, if $P_{e S}$ is very small, the surfactant in solution diffuses very rapidly, leading to a more homogeneous surfactant distribution at all times. This is illustrated in Figure S2 in Supporting Information. When $\mathrm{P}_{\mathrm{es}}=0.1$, the distribution is almost totally flattened. 

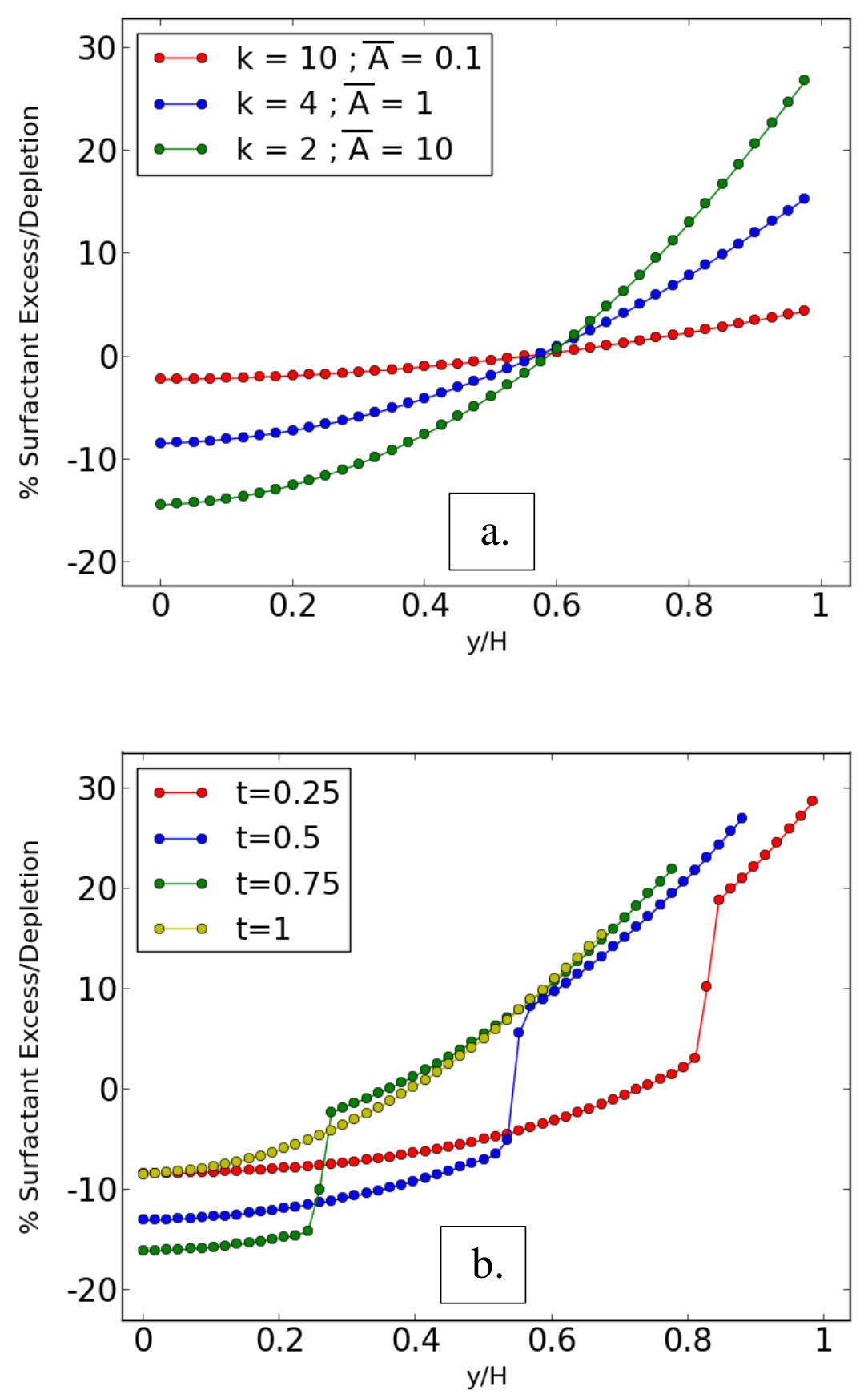

Figure 3. Percent surfactant excess/depletion as a function of scaled distance from the substrate in the vertical direction. Upward vertical convection of surfactant taken into account. $\mathrm{P}_{\mathrm{eP}}=50 ; \mathrm{P}_{\mathrm{eS}}=0.5 ; \mathrm{t}=1$ at the end of the first drying step.

a. Surfactant distributions at $\mathrm{t}=1$ for different polymer/surfactant systems.

b. Evolution of the surfactant distribution at different times for an intermediate polymer/surfactant system: $\mathrm{k}=4 ; \overline{\mathrm{A}}=1$. 


\subsection{Influence of Particle Deformation. Capillary Deformation}

After a certain drying time, particles start deforming. In this simulation, deformed particles are considered as truncated spheres. The area of the polymer / water interface decreases. The way the quantitative dependence of the area on the polymer volume fraction was established, is indicated in Section 2. The previously adsorbed surfactants desorb in the water phase, increasing their mobility and the sensitivity of the distributions to convection by water. The equilibrium between adsorbed and dissolved surfactant is still considered as being reached instantaneously. A new parameter has to be taken into account, $\bar{\lambda}$, introduced by Routh and Russel ${ }^{3}$ and representing the evaporation rate divided by the deformation rate.

If $1<\bar{\lambda}<100$, particles deform by the well-known capillary deformation mechanism. ${ }^{4} \mathrm{~A}$ value of 25 was chosen. In this case, deformation starts once the system has reached close packing throughout the film thickness. Results are shown in Figure 4. With a $\mathrm{P}_{\mathrm{eP}}$ of 50, a sharp particle front between the wet gel and the fluid zone is expected and observed indeed (Fig. 4a). After close packing, deformation occurs uniformly through the film thickness, resulting in particle distributions represented as horizontal lines moving upward as drying and deformation proceeds. ${ }^{17}$ The simulation was stopped at time 1.49, when the particle volume fraction had reached $\sim 0.9$. [Dommage que ça n'aille pas plus haut. Vrai aussi pour les parties suivantes. On va nous demander pourquoi. Ne vaudrait-il pas mieux anticiper la question et y répondre dès la première soumission ?]

Figure $4 \mathrm{~b}$ is of course identical to Fig. $3 \mathrm{~b}$ until $\mathrm{t}=1$. After starting deformation, the surfactant is progressively desorbed in water and the downward deformation of the gel pushes water and surfactant upward. As a result, the distribution is more and more distorted in favor of the surface side. At the particle volume fraction of 0.9 , the surface excess is almost $30 \%$ and the depletion on the substrate is less than $-10 \%$.

At this point, let us discuss the issue of surfactant desorption associated to particle packing and deformation. In this study, we only took into account the decrease of the number of adsorption sites associated to the reduction of the area of the polymer / water interface upon deformation. However, the surfactant could (at least to a certain extent) desorb before particles come in contact, essentially because of the repulsion between charged polar heads. This would enhance the surfactant mobility and probably affect the final distributions. On the other hand, it is also possible that the surfactant remains trapped between particles during the deformation process.

The phenomenon of surfactant desorption from the particle surfaces upon deformation is

mentioned in most of the papers dealing with surfactant distributions in latex films. ${ }^{7}, 30,9,10$ However, only few report direct experimental evidence of desorption. Kientz et al.$^{31}$ followed the kinetics of surfactant desorption from a deforming array of packed particles through $\mathrm{Na}$ titration by atomic absorption spectroscopy, showing also the decreased mobility of the desorbed surfactant when particle volume fraction reaches high values, around $90 \%$. Belaroui et al. studied desorption of deuterated SDS by SANS. ${ }^{32}$ They showed that desorption occurred at a particle volume fraction of $80 \%$ and that a small fraction of SDS remained trapped between deformed particles. 
There is an obvious lack of precise and general, experimental and theoretical knowledge of desorption and fate of desorbed surfactant in latex films. This was also stressed by Keddie and Routh in the conclusion of the chapter devoted to surfactant distribution in their book on latex film formation. ${ }^{4}$ 

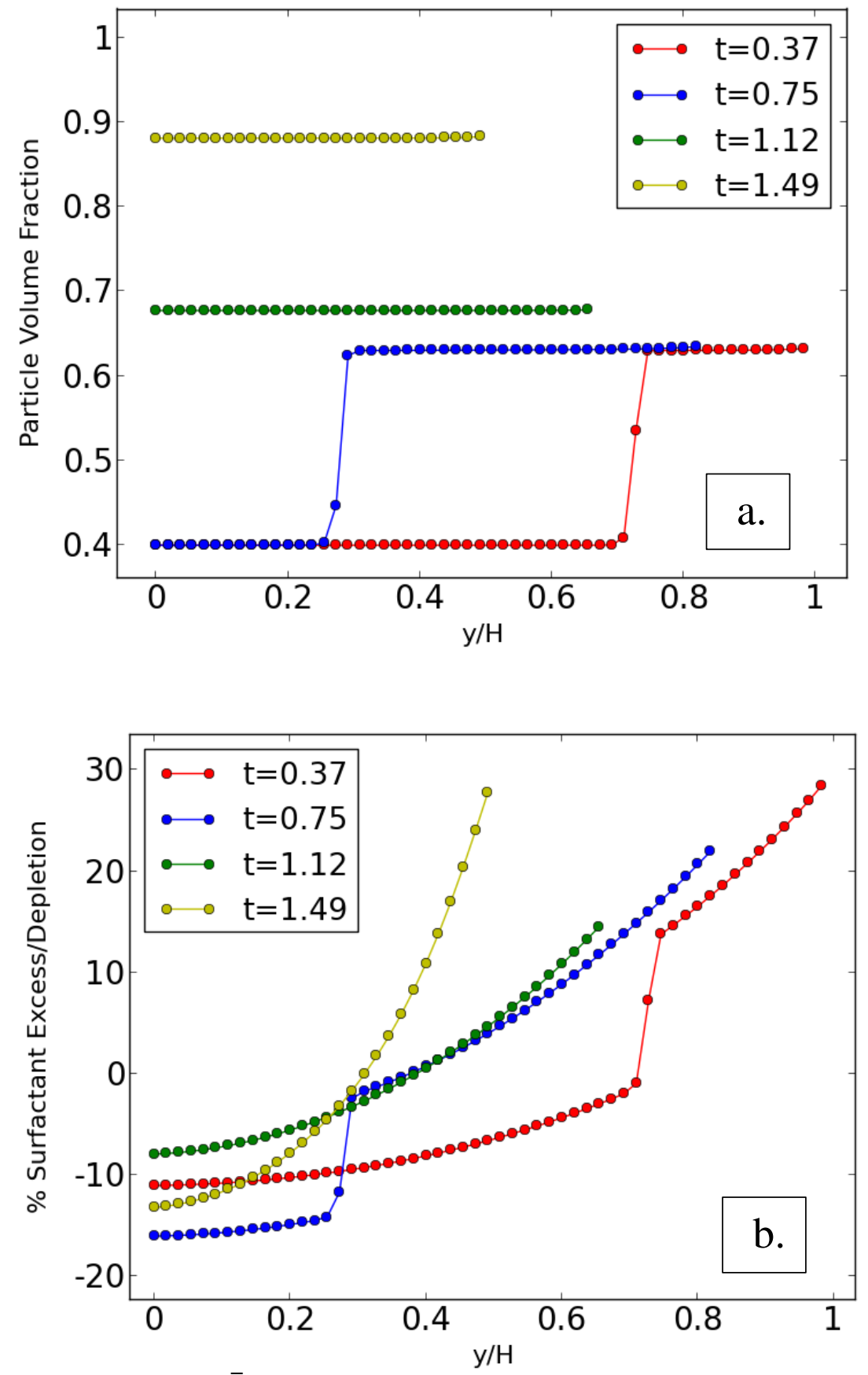

Figure 4. Capillary deformation. $\bar{\lambda}=25$.

$\mathrm{P}_{\mathrm{eP}}=50 ; \mathrm{P}_{\mathrm{eS}}=0.5 ; \mathrm{t}=1$ at the end of the first drying step.

a. Particle volume fraction versus scaled distance from the substrate at various times.

b. Corresponding surfactant distributions. $\mathrm{k}=4 ; \overline{\mathrm{A}}=1$. 


\subsection{Influence of Particle Deformation. Wet Sintering and Skin Formation}

When $\bar{\lambda}$ is less than 1 , drying becomes slower than deformation. This happens with soft particles. Once particles get in contact, they start losing their spherical shape in presence of water. This mechanism is called wet sintering. ${ }^{4}$ The driving force for wet sintering is the polymer / water interfacial tension. An elegant model, based on the different curvatures and the corresponding Laplace pressures applied on a couple of coalescing particles, was proposed by Bradford and Vanderhoff ${ }^{33}$ as early as 1966 to account for the fact that particles could be deformed despite of the usually low values of the polymer / water interfacial tension. The possibility to significantly deform particles by wet sintering was experimentally confirmed by Dobler et al. ${ }^{34}$ Furthermore, when $\mathrm{P}_{\mathrm{eP}}$ is high (our case), particles accumulate at the film surface and start deforming while the rest of the film below is still totally fluid. One speaks of "skin formation" in this situation. ${ }^{35}$ It has the consequence of reducing the drying rate ${ }^{36}$ but this effect was not taken into account at this stage. $\bar{\lambda}$ was set at 0.5 in this part (see Supporting Information for other values). The time scale is the same as in the preceding sections but the state of the system at $t=1$ is now different. $t=1$ still corresponds to the time when the particle volume fraction has reached 0.64 at the level of the substrate (like before). However, on the air side, deformation has already started and the particle volume fraction is well above 0.64 (see Fig. 5a).

Figure $5 \mathrm{a}$, showing the particle volume fraction versus distance to the substrate at different times looks similar to Fig. 4a except that the gel side of the steps, or the almost straight line after $\mathrm{t}$ $=1$, present an increasing slope due to deformation by wet sintering, more pronounced closer to the surface because there close packing occurred and deformation started earlier. Corresponding surfactant distributions can be seen in Figure 5b. Again (like in Fig. 3b and 4b), steps associated to the particle front advancing toward the substrate are visible. Kinks on the gel side appear at the points where particle volume fraction reaches 0.64 . However, after $t=1$, the distribution changes radically: instead of an excess at the surface, there is now an excess at the substrate side, with a shallow minimum slightly under the surface. This is obviously linked to the change in the particle deformation mechanism, from capillary deformation to wet sintering and skin formation.

As deformation progresses in the skin, water (plus surfactant) is expelled downwards. To understand this, let us imagine one horizontal layer of particles in contact and immersed in water. Wet sintering creates contact zones of increasing size between adjacent particles. The polymer pushes water away from the contact zone to take the place, equally up and down. If now several layers in contact are piled up, upper ones are more deformed than lower ones and, at a given level, the upward flow is hindered by the lower permeability of the upper layers, resulting in a neat downward flow. A similar reasoning was used by Guigner et al. ${ }^{37,38}$ to explain the clearly observed downward flux of water and surfactant in a poly(dimethyl siloxane) emulsion deforming and coalescing from top to bottom. Another way of thinking is to consider a gel zone several layers of particles in contact thick, the top layer just below the level of water, deforming with a gradient of deformation (more deformation on top than on bottom). This would correspond to an upward contraction of the gel, and, correlatively, a downward motion of water.

To understand the distributions in Fig. 5b, there are now two opposite water fluxes to consider: the upward flux associated to the downward moving particle front, dominating at times 
below 1, and the downward flux due to the particle deformation gradient by wet sintering, dominating at times above 1 .
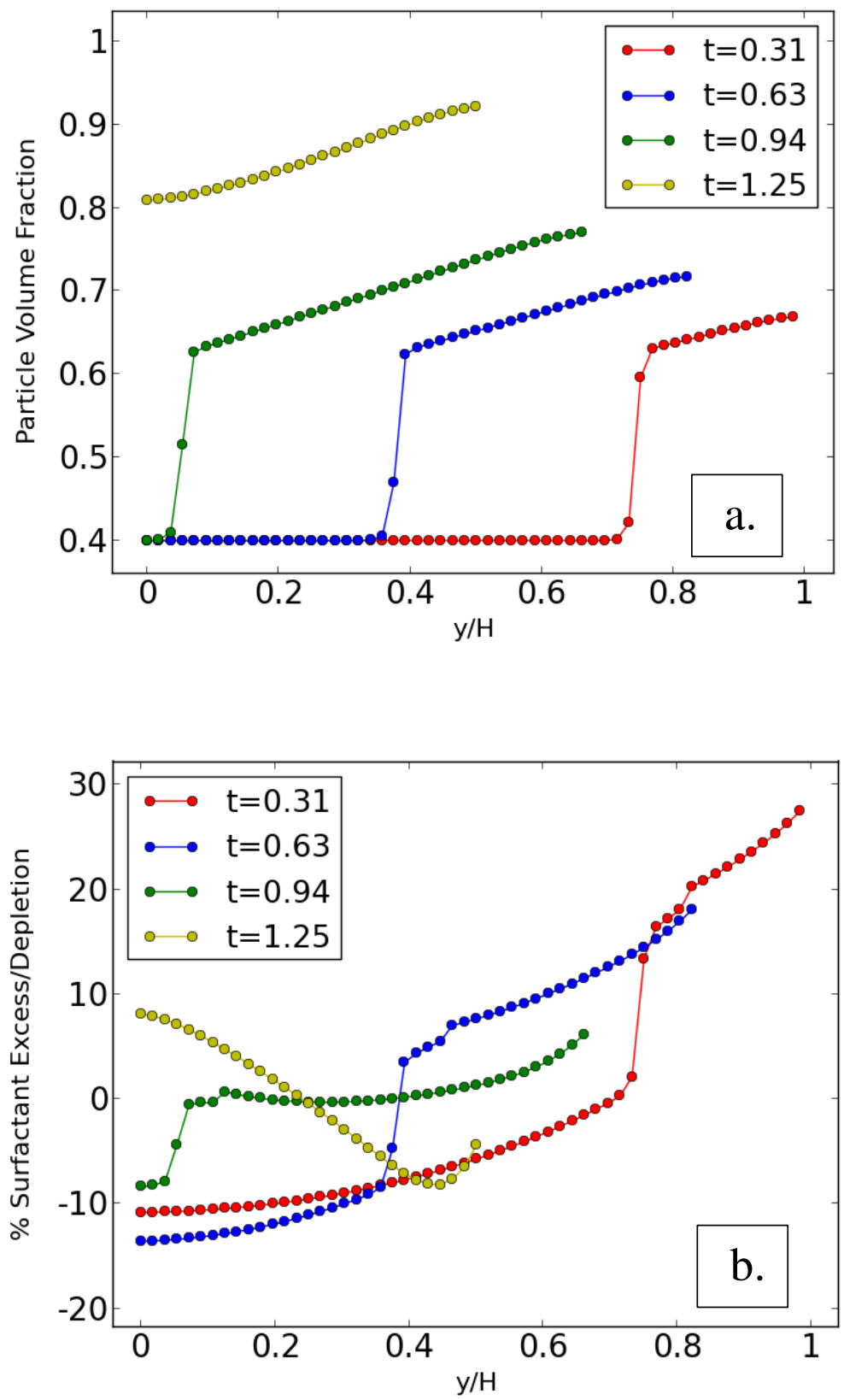

Figure 5. Deformation by wet sintering. $\bar{\lambda}=0.5$.

$\mathrm{P}_{\mathrm{eP}}=50 ; \mathrm{P}_{\mathrm{eS}}=0.5 ; \mathrm{t}=1$ when particle volume fraction has reaches 0.64 in contact with the substrate.

a. Particle volume fraction versus scaled distance from the substrate at various times.

b. Corresponding surfactant distributions. $k=4 ; \bar{A}=1$. 
A peculiarity in the surfactant distribution at $t=1.25$ (Fig. 5b) is the minimum located slightly under the surface. In order to better apprehend the phenomenon, more distributions were calculated between times 0.94 and 1.25 (Fig. 6a). At time $\sim 1$, there is a surfactant excess at the surface which progressively decreases while the concentration slightly below also decreases, creating and deepening a minimum in the distribution. The downward flux due to wet sintering decreases the surfactant concentration on the surface side but the initial surface excess associated to the previous upward flux persists as deformation is continuing. This is confirmed by data in Fig. $6 \mathrm{~b}$ showing distributions at $\mathrm{t}=1.25$ for different polymer / surfactant systems. When there is more surfactant in the water phase (case (ii), $\mathrm{k}=2, \overline{\mathrm{A}}=10$ ), the initial surfactant excess is more pronounced (Fig. 3a) and more persistent upon particle deformation.

In order not to lengthen the article too much, some details were withdrawn from the main text and switched to the Supporting Information Section. Figure S3 is a complement to Figure 6. It shows surfactant distributions at various times for the intermediate polymer / surfactant system ( $\mathrm{k}$ $=4 ; \overline{\mathrm{A}}=1)$ when Peclet numbers are reduced $\left(\mathrm{P}_{\mathrm{eP}}=5 ; \mathrm{P}_{\mathrm{eS}}=0.1\right)$. In this case, a particle front still exists but it is much more diffuse and consequently the upward water flux associated to the downward moving particle front is much weakened. As a result, only the downward water flux is really operative: the excess at the substrate side is more pronounced and there is no minimum in the curve below the surface. It has also to be noted that, in this situation, curves are not flattened despite of the small surfactant Peclet number (0.1).

Figure S4 complements Figure 6a. In the same conditions, it shows how the surfactant is partitioned between the particle surfaces and the water phase. At time $\sim 1$, about $30 \%$ of the total surfactant is in the water phase. As deformation progresses, this percentage progressively increases due to surfactant desorption, to reach around $50 \%$ at $t=1.25$. Curves also show that the percentage is higher on the surface side where deformation and desorption is more pronounced.

Finally, Figure S5 shows the extreme sensitivity of the surfactant distributions to the precise value of $\bar{\lambda}$ in the wet sintering regime. It compares distributions at $t=1.25$ in our reference system $\left(\mathrm{k}=4 ; \overline{\mathrm{A}}=1 ; \mathrm{P}_{\mathrm{eP}}=50 ; \mathrm{P}_{\mathrm{eS}}=0.5\right)$ for three values of $\bar{\lambda}: 0.35 ; 0.5$ (like in Fig. 6$)$ and 0.9 . The effect is dramatic: at $\bar{\lambda}=0.35$ the dissymmetry of the distribution in favor of the substrate side is massive $(+35 \%$ at the substrate; $-55 \%$ at the surface $)$ whereas at $\bar{\lambda}=0.9$ the result is reversed $(-10 \%$ at the substrate; $+20 \%$ at the surface). Such kind of result would be extremely difficult to infer from the values of the parameters just by thinking. The help of a simulation tool is essential. 

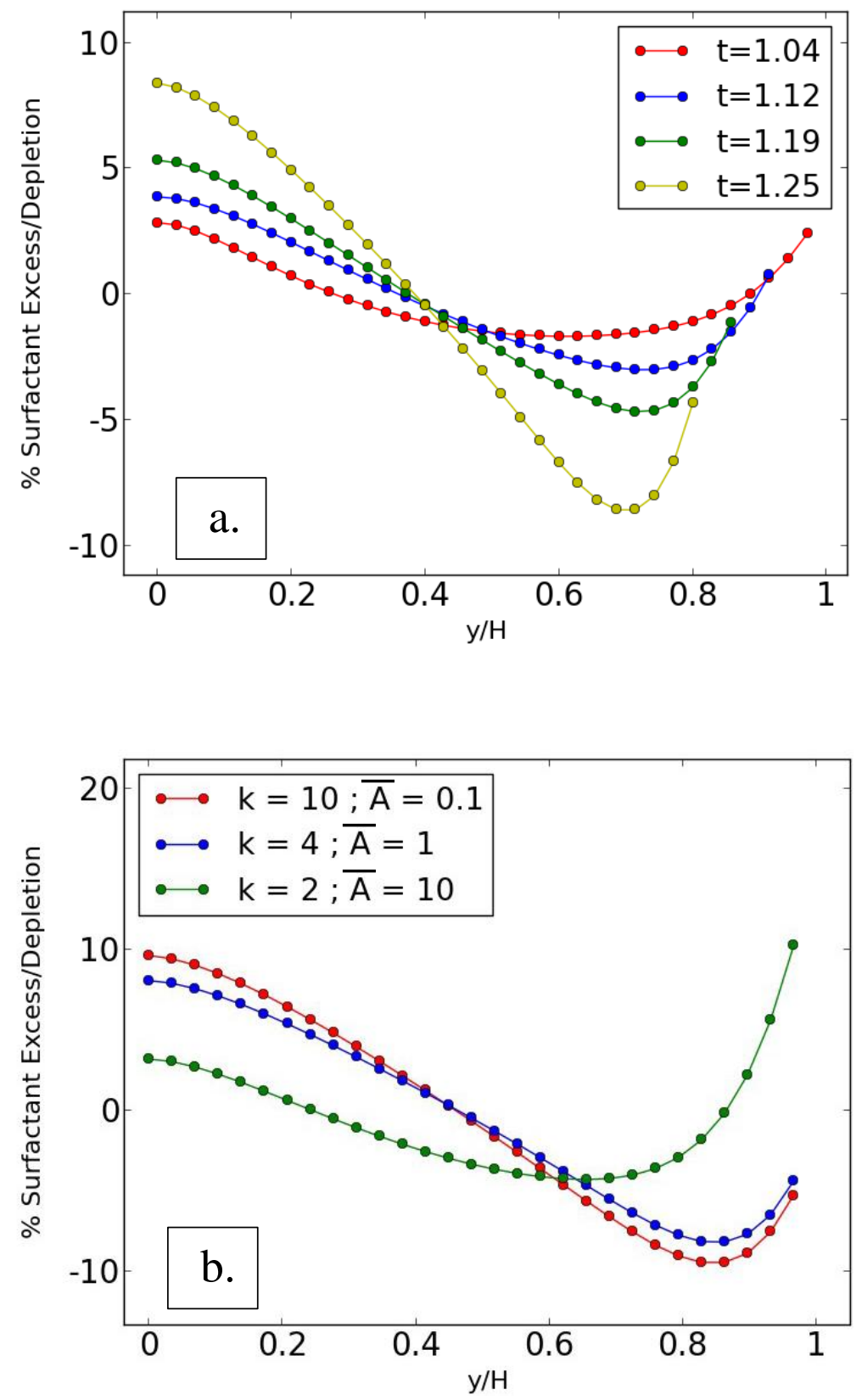

Figure 6. Deformation by wet sintering. $\bar{\lambda}=0.5$. Percent surfactant excess/depletion as a function of scaled distance from the substrate in the vertical direction.

$\mathrm{P}_{\mathrm{eP}}=50 ; \mathrm{P}_{\mathrm{eS}}=0.5 ; \mathrm{t}=1$ when particle volume fraction has reached 0.64 in contact with the substrate.

a. Intermediate polymer / surfactant system $(\mathrm{k}=4 ; \overline{\mathrm{A}}=1)$ at various times

b. Different polymer / surfactant systems (as indicated) at $\mathrm{t}=1.25$. 


\subsection{Influence of Particle Deformation. Receding Water Front}

According to the Routh and Russel's ${ }^{3}$ deformation map, when $\bar{\lambda}$ is comprised between $10^{2}$ and $10^{4}$, deformation occurs in the "Receding Water Front" regime, intermediate between capillary deformation and dry sintering. It means that capillary deformation is not fast enough compared to drying, and a drying front (an air / water interface) penetrates into the film and sweeps over the particle array. Underneath the drying front, the capillary mechanism still operates whereas above further deformation continues through dry sintering. The reality of dry sintering was demonstrated by Keddie et al. ${ }^{39}$

A value of 200 was taken for $\bar{\lambda}$. Our first assumption is that, as the receding water front passes through the gel, the surfactant which is adsorbed at the particle surface is not desorbed. Our second assumption concerns what happens to the surfactant in solution. Two different scenarios were examined: either unadsorbed surfactant remains in solution, or it is deposited onto the gel, with the deposited quantity equal to the previously adsorbed amount. Results are presented in Figure 7. [La distribution des particules serait intéressante à montrer aussi.] Depending on the scenario, at the end of the simulation run (at $\mathrm{t}=1.49$ ), a marked surfactant excess is found either at the substrate, or at the surface. Remember that, at $\mathrm{t}=1$ (end of first drying step), there is an excess at the surface and a depletion at the substrate (Fig. 3a). This distribution is totally changed after further drying and deformation. Figure 7 a shows the distributions in the first case (surfactant remaining in water). The water front is at the level of the discontinuities. The region below the front is more and more enriched with surfactant, leaving a depleted dry gel. However, when approaching the substrate, the concentration in the dry part increases progressively because, the solution being more and more concentrated, more surfactant is adsorbed at the polymer surface, in accordance with the isotherm which applies to the system.. The decrease of the concentration toward the substrate in the wet region is due to the accumulation of surfactant just below the drying front. With $\mathrm{P}_{\mathrm{es}}=0.5$, diffusion is not important enough to redistribute the surfactant. It is also likely that the surfactant mobility decreases progressively in the more and more deformed gel, leading to a decrease of the diffusion coefficient and an increase of the real $\mathrm{Pes}_{\mathrm{es}}$.

Opposite results appear in Figure $7 \mathrm{~b}$ (surfactant deposited). The downward moving water front is also clearly visible. Now, as the water front moves down, the tendency is to decrease the concentration both in the dry and in the wet gel. This is an obvious consequence of surfactant deposition. In the wet region, toward the substrate, concentration increases, for the same reason as it decreases in Fig. 7a. Just below the front, deposition has created a depletion which cannot be reequilibrated by diffusion.

Results are strongly dependent on the assumptions about the behavior of the surfactant with respect to the water front. All these assumptions are questionable. Opposite to our first assumption above, it is not impossible that the adsorbed surfactant would be desorbed (at least partially) by the water front in order to stay in water, leaving a naked polymer surface that might have a lower surface tension with air than if the surfactant was stayed present. This phenomenon, in conjunction with the convection of the surfactant in solution toward the substrate (the assumption leading to Fig. 7a), was invoked in the model developed by Arnold et al. ${ }^{11}$ On the other hand, deposition is possible but perhaps differently than what is proposed here (just doubling the adsorbed amount). Tzitzinou et al. ${ }^{9}$ insist on the term deposition but, in their sense, it only concerns the surfactant 
deposited on top of the particle array. And, again in the article by Arnold et al. ${ }^{11}$, it is proposed that surfactant is periodically deposited in large quantity when the water front is no longer able to drag it further down. Clearly, the answers to these questions are not included in the five parameters involved in this simulation. A refinement of the simulation, inspired by specific experiments on this issue, is required to be more conclusive in the case of receding water front. 

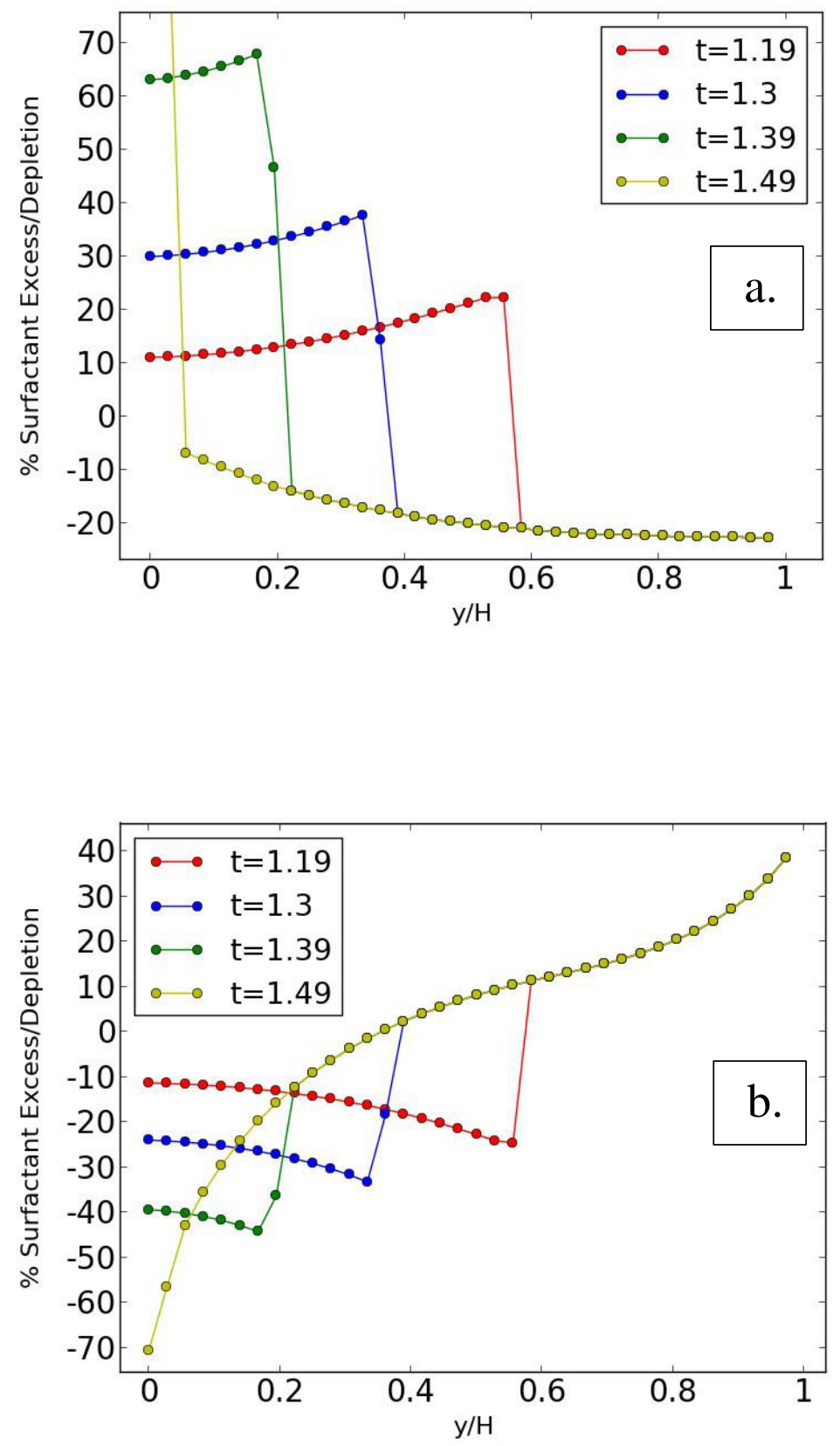

Figure 7. Deformation by receding water front. $\bar{\lambda}=200$. Percent surfactant excess/depletion as a function of scaled distance from the substrate in the vertical direction.

$\mathrm{P}_{\mathrm{eP}}=50 ; \mathrm{P}_{\mathrm{eS}}=0.5 ; \mathrm{k}=4 ; \overline{\mathrm{A}}=1 ; \mathrm{t}=1$ when particle volume fraction has reached 0.64 in contact with the substrate.

a. Surfactant in solution is totally swept down by the drying front.

b. Surfactant in solution is partially deposited onto the particle array by the drying front. 


\section{CONCLUSIONS}

Owing to its marked influence on major final properties like permeability and adhesion, surfactant distribution in latex films has received much attention in the literature. However, most studies are experimental. Our simulation is a contribution to the topic, on the theoretical side. Our approach is inspired by cellular automata ${ }^{18}$ and, as far as specific equations are concerned, it is largely beholden to the work of Routh and coworkers, ${ }^{3,14,15}$ while going further toward the complete film formation process. There are five parameters in the simulation. Two $(k$ and $\bar{A})$ depend on the nature of the polymer / surfactant system, two $\left(\mathrm{P}_{\mathrm{eP}}\right.$ and $\left.\mathrm{P}_{\mathrm{eS}}\right)$ depend on the film formation conditions and on the sizes of the polymer and surfactant; and one $(\bar{\lambda})$ depends on the viscoelastic properties of the polymer and on the drying rate. The complex interplay between those parameters leads to contrasted surfactant distribution results that would be essentially unpredictable just by thinking. It was shown that during the drying step, convection of the surfactant by water plays an important role. The surfactant distribution at the end of the first drying step is largely altered during the particle deformation step in a way that much depends on the particular deformation mechanism. In the case of capillary deformation, the pre-existing excess at the surface side is much amplified, whereas, if wet sintering and skin formation occur, the dissymmetry of the distribution is reversed in favor of the substrate side. In the case of the receding water front regime, the results, large excess on one side of the film or the other, depend on the interaction of the surfactant with the water front. The lack of knowledge in the literature on this particular point impedes definitive conclusions.

All simulation works require experimental validation. The distributions presented here are in qualitative agreement with experimental results in the literature. Surfactant excesses at the surface side were very often mentioned and are reproduced by simulation, in standard conditions. It is the same for excesses at the substrate side, they were shown in experimental studies and are reproduced by simulation, for instance in the case of wet sintering and skin formation. It is difficult to be more quantitative because from the description of published experiments, it is very difficult, if not impossible, to deduce numerical values of the required simulation parameters. For precise quantitative validations, specific experiments, in well controlled conditions, should be designed. At the present stage, the simulation is not yet able to reproduce the well documented surfactant aggregate formation in the bulk of the film. Even more difficult would be to account for the structuration of the surfactant deposited or exudated at the top surface of the film.

There are thus several ways of improvement for this simulation. An interesting point would be to take into account micelle containing systems. It would also have the advantage of opening the way to more complicated systems containing small charged objects like nanoparticles. The main refinement we are thinking of is to increase the dimensionality of the treatment, from 1D to 2D and ultimately to 3D. A 2D approach in the plane of the film is in progress, shedding light on the difficult question of what happens when two drying fronts moving in different directions get close and interfere. 3D simulations will start raising serious computing problems but that should not be intractable. The ideal way of working would be to push forward the simulation in parallel with specifically conducted experiments, each approach taking advantage of the other, for example about the problem of surfactant desorption and interaction with a moving drying front.

In spite of the mentioned limitations, we believe that this simulation is already versatile 
enough to be able to constitute a help to practitioners in industry, formulating complicated systems like paints or cosmetics. On the other hand, this kind of work, questioning again the complex film formation process, might be of interest to academic scientists in the field of colloid drying and, more generally, of colloidal physical chemistry.

\section{ASSOCIATED CONTENT}

\section{Supporting Information}

1. Comparison with the Model of Gundabala et al.

2. Effect of Surfactant Peclet Number in the First Drying Step

3. Supplements to the part dealing with wet sintering $(\bar{\lambda}=0.5)$

4. Derivation of the transport equations by means of a variational principle

\section{ACKNOWLEDGEMENTS}

The financial support from the French National Research Agency (ANR) (Project reference: ANR11-RMNP-009-SPPLIF-5A0374) is gratefully acknowledged. 


\section{REFERENCES}

(1) Routh, A. F. Drying of Thin Colloidal Films. Rep. Prog. Phys. 2013, 76, 46603.

(2) Russel, W. B. Mechanics of Drying Colloidal Dispersions: Fluid/Solid Transitions, Skinning, Crystallization, Cracking, and Peeling. AIChE J. 2011, 57, 1378-1385.

(3) Routh, A.F.; Russel, W.B. A Process Model for Latex Film Formation: Limiting Regimes for Individual Driving Forces. Langmuir 1999, 15, 7762-7773.

(4) Keddie, J.; Routh, A. Fundamentals of Latex Film Formation: Processes and Properties; Springer: Dordrecht. The Netherlands, 2010.

(5) van der Kooij, H.M.; van de Kerkhof, G.T.; Sprakel, J. A echanistic View of Drying Suspension Droplets. Soft Matter 2016, 12, 2858-2867.

(6) Hellgren, A.C.; Weissenborn, P.; Holmberg, K. Surfactants in Water-Borne Paints. Prog. Org. Coat. 1999, 35, 79-87.

(7) Kientz, E.; Holl, Y. Distribution of Surfactants in Latex Films. Colloid Surf. A 1993, 78, $255-$ 270.

(8) Vandezande, G.A.; Rudin, A. Film Formation of Vinyl Acrylic Latexes; Effects of Surfactant Type, Water and Latex Particle Size. J. Coat. Technol. 1996, 68 (860), 63-73.

(9) Tzitzinou, A.; Jenneson, P.M.; Clough, A.S.; Keddie, J.L.; Lu, J.R.; Zhdan, P.; Treacher, K.E.; Satguru, R. Surfactant Concentration and Morphology at the Surface of Acrylic Latex Films. Prog. Org. Coat. 1999, 35, 89-99.

(10) Mallégol, J.; Gorce, J.P.; Dupont, O.; Jeynes, C.; McDonald, P.J.; Keddie, J.L. Origins and Effects of a Surfactant Excess Near the Surface of Waterborne Acrylic Pressure Sensitive Adhesives. Langmuir 2002, 18, 4478-4487.

(11) Arnold, C.; Thalmann, F.; Marques, C.; Marie, P.; Holl, Y. Surfactant Distribution in Waterborne Acrylic Films. 1. Bulk Investigation. J. Phys. Chem. B 2010, 114, 9135-9147

(12) Guyot, A.; Tauer, K. Reactive Surfactants in Emulsion Polymerization. Adv. Polym. Sci. 1994, $111,43-65$.

(13) Aramendia, E.; Mallégol, J.; Jeynes, C.; Barandiaran, M.J.; Keddie, J.L.; Asua, J.M. Distribution of Surfactants Near Acrylic Latex Film Surfaces: A Comparison of Conventional and Reactive Surfactants (Surfmers). Langmuir 2003, 19, 3212-3221.

(14) Gundabala, V.R.; Zimmerman, W.B.; Routh, A.F. A Model for Surfactant Distribution in Latex Coatings. Langmuir 2004, 20, 8721-8727.

(15) Gundabala, V.R.; Routh, A.F. Predicting Surfactant Distribution in Dried Latex Films. in Film Formation; Process and Morphology. Provder, T.; Ed. ACS Symposium Series 941. American Chemical Society, Washington, DC. 2006, 53-65.

(16) Lee, W.P.; Gundabala, R.; Akpa, B.S.; Johns, M.L.; Jeynes, C.; Routh, A.F. Distribution of Surfactants in Latex Films: A Rutherford Backscattering Study. Langmuir 2006, 22, 5314-5320.

(17) Gromer, A.; Nassar, M.; Thalmann, F.; Hébraud, P.; Holl, Y. Simulation of Latex Film Formation Using a Cell Model in Real Space: Vertical Drying. Langmuir 2015, 31, 10983-10994. 
(18) Chopard, B.; Droz, M. Cellular Automata Modeling of Physical Systems. Cambridge University Press, 1998.

(19) Routh, A.F.; Zimmerman, W.B. Distribution of Particles during Solvent Evaporation from Films. Chem. Eng. Sci. 2004, 59, 2961-2968.

(20) Lissant, K.J. The geometry of High Internal Phase Ratio Emulsions. J. Colloid Interface Sci. 1966, 22, 462-468.

(21) Princen, H.M. Pressure/Volume/Surface Area Relationships in Foams and Highly Concentrated Emulsions: Role of Volume Fraction. Langmuir 1988, 4, 164-169.

(22) Langmuir, I. The constitution and Fundamental Properties of Solids and Liquids. J. Am. Chem. Soc. 1916, 38, 2221-2295.

(23) Lam, S.; Hellgren, A.C.; Sjobërg, M.; Holmberg, K.; Schoonbrood, H.A.; Unzué, M.J.; Asua, J.M.; Tauer, K.; Sherrington, D.C.; Goni, A.M. Surfactants in Heterophase Polymerization: a Study of Film Formation Using AFM. J. Appl. Polym. Sci. 1997, 66, 187-198.

(24) Vale, H.M.; McKenna, T.F. Adsorption of Sodium Dodecyl Sulfate and Sodium Dodecyl Benzenesulfonate on Poly(vinyl chloride) Latexes. Colloids Surf. A. 2005, 268, 68-72.

(25) Arnold, C.; Klein, G.; Maaloum, M.; Ernstsson, M.; Larsson, A.; Marie, P.; Holl, Y. Surfactant Distribution in Waterborne Acrylic Films 2. Surface Investigation. Colloids Surf. A. 2011, 374, 58 68.

(26) Vijayendran, B.R.; Bone, T.; Gajria, C. Surfactant Interactions in Poly(vinyl acetate) and Poly(vinyl acetate-butyl acrylate) Latexes. J. Appl. Polym. Sci. 1981, 26, 1351-1359.

(27) Bindschaedler, C.; Gurny, R.; Doelker, E. Influence of Emulsifiers on Film Formation from Cellulose Acetate Latexes: Experimental Study of Phase Separation Phenomena Due to Sodium Dodecyl Sulfate. J. Appl. Polym. Sci. 1987,34, 2631-2647.

(28) Bindschaedler, C.; Gurny, R.; Doelker, E. Influence of Emulsifiers on Film Formation from Cellulose Acetate Latexes. Modeling Approach to the Fate of Emulsifiers in Highly Plasticized Films. J. Appl. Polym. Sci. 1989,37, 173-182.

(29) Evanson, K.W.; Thorstenson, T.A.; Urban, M.W. Surface and Interfacial FTIR Spectroscopic Studies of Latexes. II. Surfactant - Copolymer Compatibility and Mobility of Surfactants. J. Appl. Polym. Sci. 1991, 42, 2297-2307.

(30) Juhué, D.; Wang, Y.; Lang, J.; Leung, O.M.; Goh, M.C.; Winnik, M.A. Surfactant Exudation in the Presence of a Coalescing Aid in Latex Films Studied by Atomic Force Microscopy. J. Polym. Sci. Part B. Polym. Phys. 1995, 33, 1123-1133.

(31) Kientz, E.; Dobler, F.; Holl, Y. Desorption of the Surfactant from the Particle Surface during Latex Film Formation. Polym. Intern. 1994, 34, 125-134.

(32) Belaroui, F.; Cabane, B.; Dorget, M.; Grohens, Y.; Marie, P.; Holl, Y. Small-Angle Neutron Scattering Study of Particle Coalescence and SDS Desorption during Film Formation from Carboxylated Acrylic Latices. J. Colloid Interf. Sci. 2003, 262, 409-417.

(33) Bradford, E.B.; Vanderhoff, J.W. Morphological Changes in Latex Films. J. Macromol. Chem. 1966, 1, 335-360. 
(34) Dobler, F.; Pith, T.; Lambla, M.; Holl, Y. Coalescence Mechanisms of Polymer Colloids. 1. Coalescence under the Influence of Particle-Water Interfacial Tension. J. Colloid Interf. Sci. 1992, $152,1-11$.

(35) Mallégol, J.; Bennett, G.; Dupont, O.; McDonald, P.J.; Keddie J.L. Skin Development During the Film Formation of Waterborne Acrylic Pressure Sensitive Adhesives Containing Tackifying Resin. J. Adhes. 2006, 82, 217-238.

(36) Erkselius, S.; Wadsö, L.; Karlsson, O.J. Drying Rate Variations of Latex Dispersions Due to Salt Induced Skin Formation. J. Colloid Interf. Sci. 2008, 317, 83-95.

(37) Guigner, D.; Fischer, C.; Holl, Y. Film Formation from Concentrated Reactive Silicone Emulsions. 1. Drying Mechanism. Langmuir 2001, 17, 3598-3606.

(38) Guigner, D.; Fischer, C.; Holl, Y. Film Formation from Concentrated Reactive Silicone Emulsions. 2. Surfactant distribution. Langmuir 2001, 17, 6419-6425.

(39) Keddie, J.L.; Meredith, P.; Jones, R.A.L.; Donald, A.M. Kinetics of Film Formation in Acrylic Latices Studied with Multiple-Angle-of- Incidence Ellipsometry and Environmental SEM. Macromolecules 1995, 28, 2673-2682. 


\title{
Simulation of Vertical Surfactant Distributions in Drying Latex Films
}

\author{
A. Gromer, ${ }^{1}$ F. Thalmann, ${ }^{1}$ P. Hébraud,${ }^{2}$ Y. Holl ${ }^{1, *}$ \\ 1. CNRS-ICS \& Université de Strasbourg \\ 23, rue du Loess BP 84047 \\ 67034 Strasbourg Cedex 2 \\ France \\ 2. IPCMS, 23 rue du Loess BP 43 \\ 67034 Strasbourg Cedex 2 \\ France \\ *yves.holl@unistra.fr
}

\section{SUPPORTING INFORMATION}

1. Comparison with the Model of Gundabala et al.

2. Effect of Surfactant Peclet Number in the First Drying Step

3. Supplements to the Part Dealing with Wet Sintering $(\bar{\lambda}=0.5)$

4. Derivation of the Transport Equations by Means of a Variational Principle 


\section{Comparison with the Model of Gundabala et al.}

Our simulation (Figures on the left) almost perfectly fits the profiles calculated by Gundabala et al., ${ }^{1,2}$ (Figures on the right. Reprinted with permission from reference 2 below. Copyright 2006 American Chemical Society) as shown in Figure S1 below.
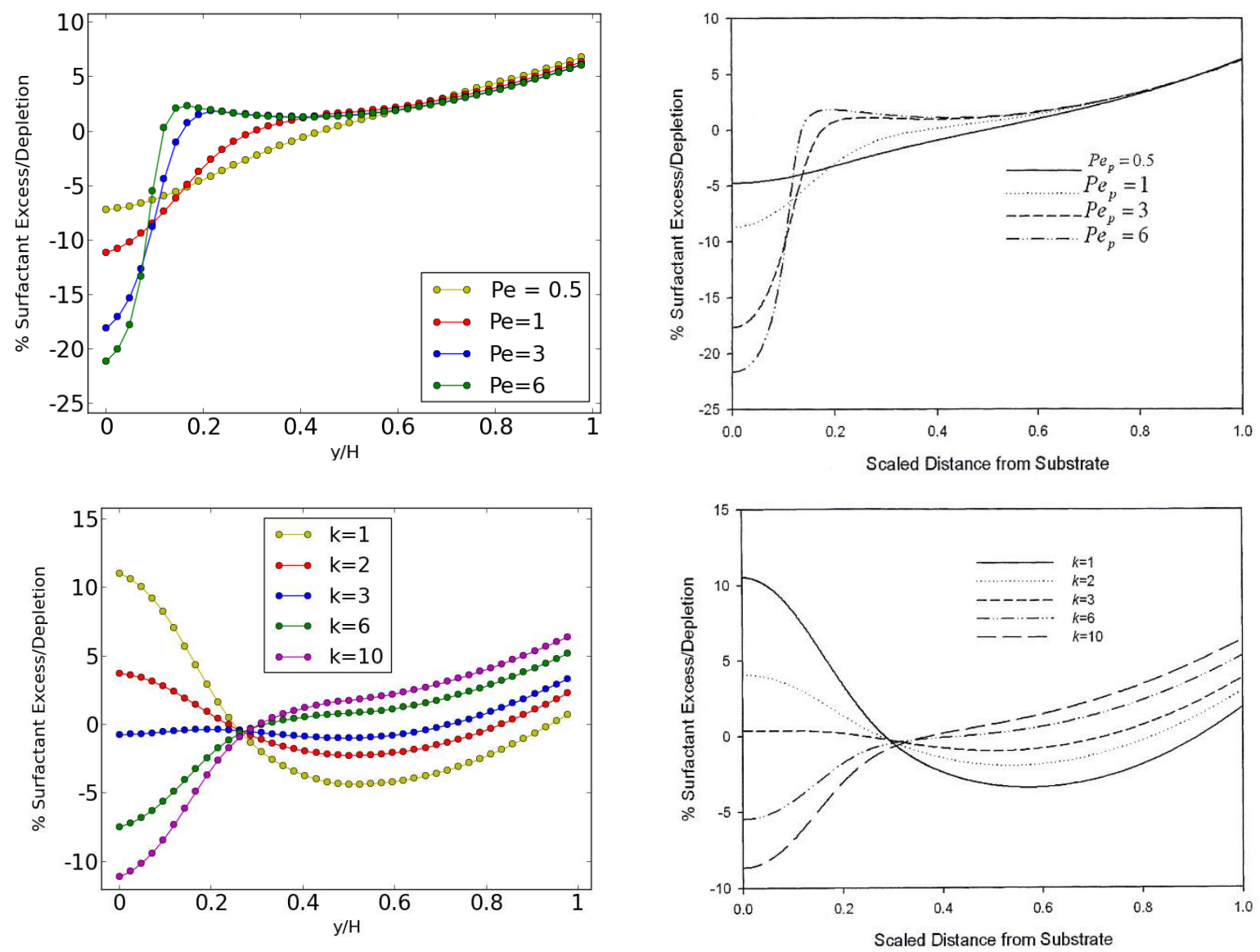

Figure S1. Surfactant distributions just before the end of the first drying stage versus distance from substrate scaled by the film thickness. Surfactant concentration is expressed in percent of excess or depletion compared to the hypothetical situation where the surfactant was distributed homogeneously throughout the film thickness.

Top line: $\overline{\mathrm{A}}=1 ; \mathrm{k}=10 ; \mathrm{Pe}_{\mathrm{S}}=0.5 ; \mathrm{Pe}$ as indicated.

Bottom line: $\overline{\mathrm{A}}=1 ; \mathrm{Pe}_{\mathrm{S}}=0.5 ; \mathrm{P} \mathrm{e}_{\mathrm{P}}=1 ; \mathrm{k}$ as indicated.

(1) Gundabala, V.R.; Zimmerman, W.B.; Routh, A.F. A Model for Surfactant Distribution in Latex Coatings. Langmuir 2004, 20, 8721-8727.

(2) Gundabala, V.R.; Routh, A.F. Predicting Surfactant Distribution in Dried Latex Films. in Film Formation; Process and Morphology. Provder, T.; Ed. ACS Symposium Series 941. American Chemical Society, Washington, DC. 2006, 53-65. 


\section{Effect of Surfactant Peclet Number in the First Drying Step}

This Figure complements Fig. 3 in the article. A small surfactant Peclet number tends to flatten the surfactant distribution.

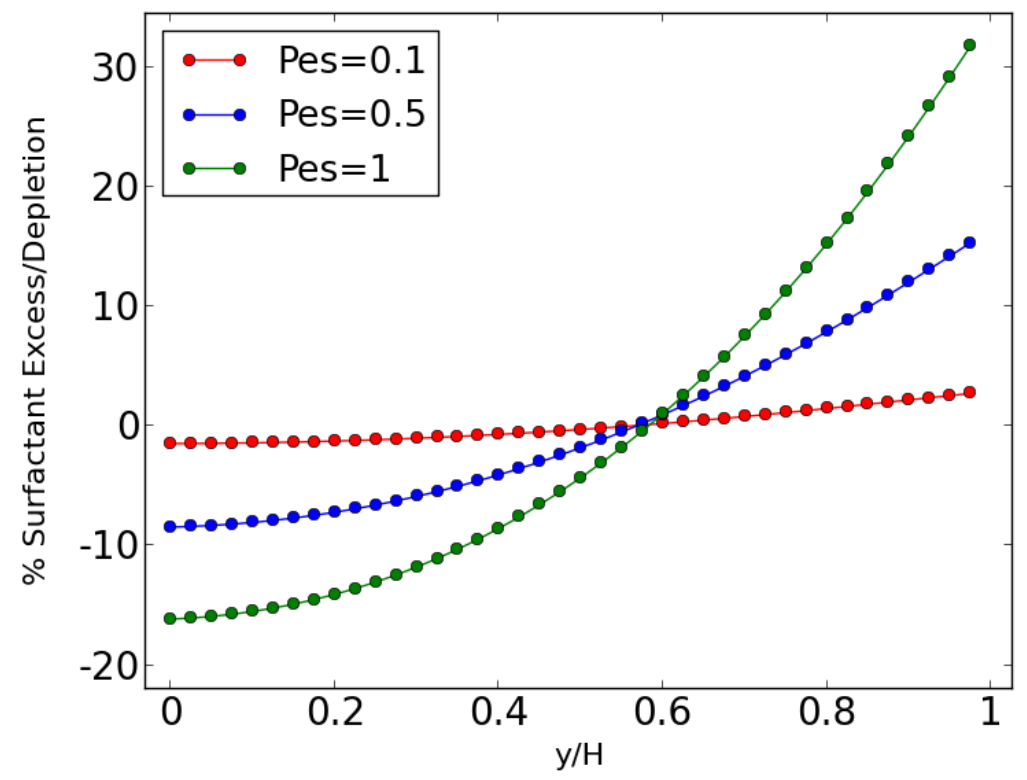

Figure S2. Percent surfactant excess/depletion as a function of scaled distance from the substrate in the vertical direction. Vertical convection of surfactant taken into account. $\mathrm{k}=4 ; \overline{\mathrm{A}}=1 ; \mathrm{P}_{\mathrm{eP}}=50 ; \mathrm{P}_{\mathrm{eS}}$ as indicated in the insert. 


\section{Supplements to the Part Dealing with Wet Sintering $(\bar{\lambda}=0.5)$}

Figure S3 is to compare to Figure 6 in the text. It shows surfactant distributions when Peclet numbers are reduced. Excesses at the substrate are enhanced and minima in the curves are no longer observed.

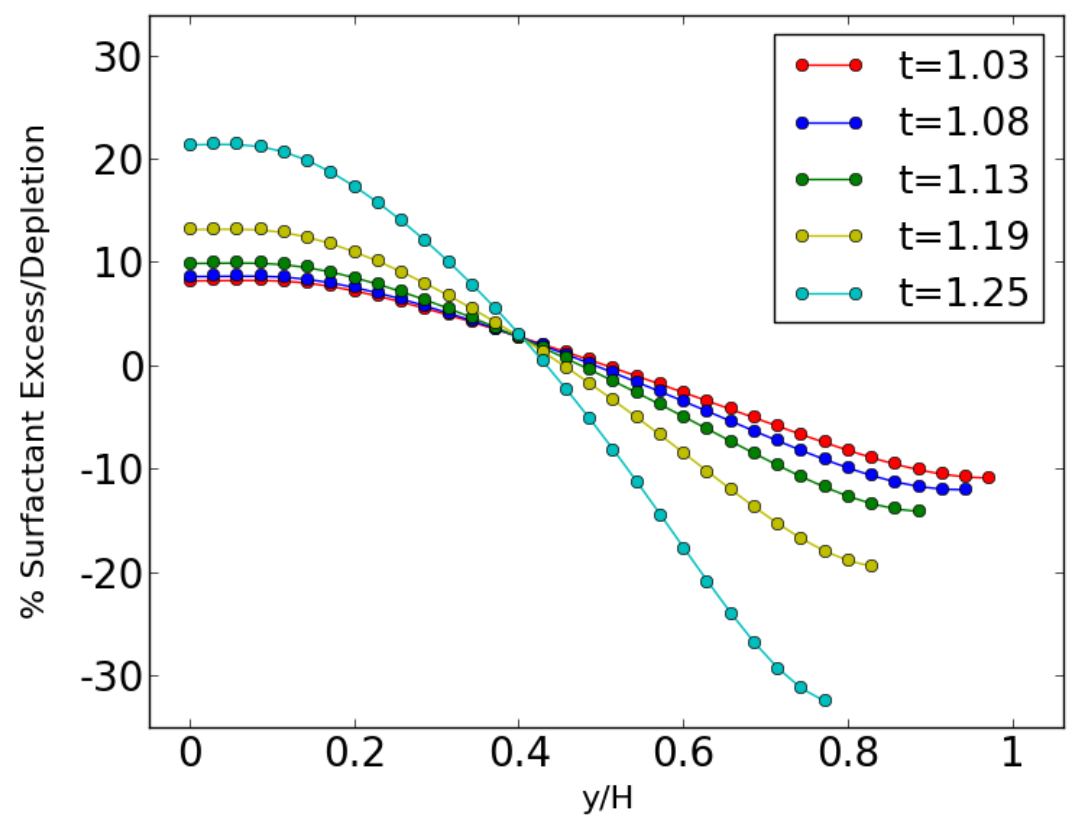

Figure S3. Deformation by wet sintering. $\bar{\lambda}=0.5$. Percent surfactant excess/depletion as a function of scaled distance from the substrate in the vertical direction.

$\mathrm{P}_{\mathrm{eP}}=5 ; \mathrm{P}_{\mathrm{eS}}=0.1 ; \mathrm{t}=1$ when particle volume fraction has reached 0.64 in contact with the substrate. Intermediate polymer / surfactant system $(\mathrm{k}=4 ; \overline{\mathrm{A}}=1)$ at various times. 
Figure S4 complements Figure 6a in the text. It shows how the surfactant is partitioned between the particle surfaces and the water phase.

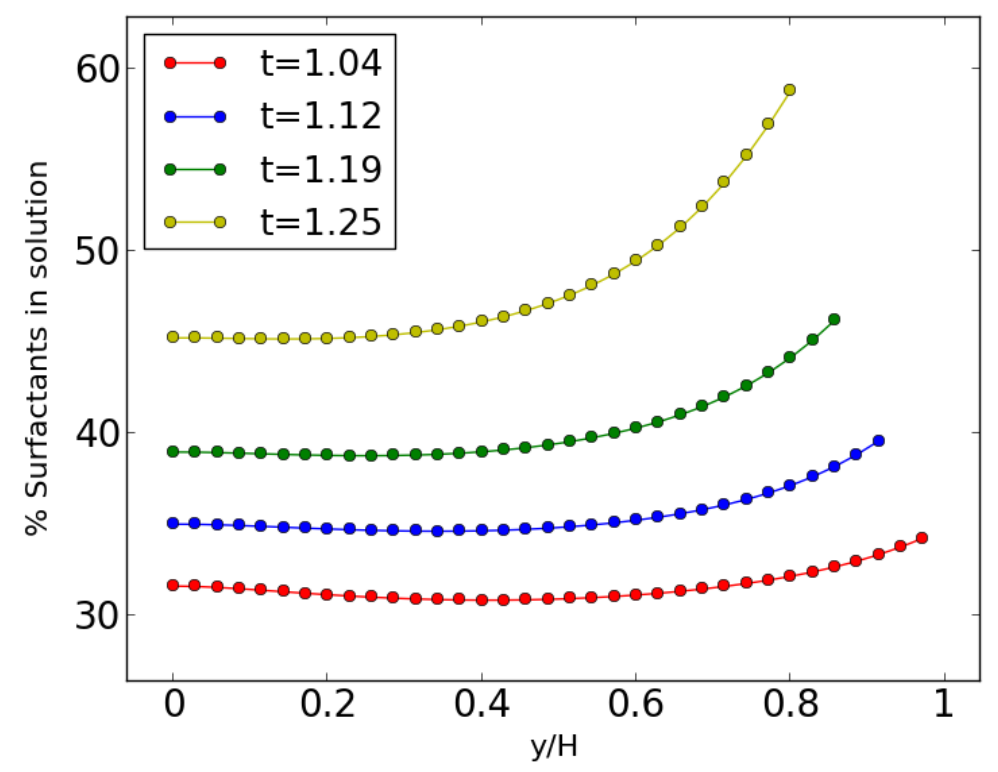

Figure S4. Deformation by wet sintering. $\bar{\lambda}=0.5$. Surfactant partitioning between particle surfaces and water phase as a function of scaled distance from the substrate in the vertical direction.

$\mathrm{P}_{\mathrm{eP}}=50 ; \mathrm{P}_{\mathrm{eS}}=0.5 ; \mathrm{t}=1$ when particle volume fraction has reached 0.64 in contact with the substrate.

Intermediate polymer / surfactant system $(\mathrm{k}=4 ; \overline{\mathrm{A}}=1)$ at various times. 
Figure S5 shows the extreme sensitivity of the surfactant distributions to the precise value of $\bar{\lambda}$ in the wet sintering regime.

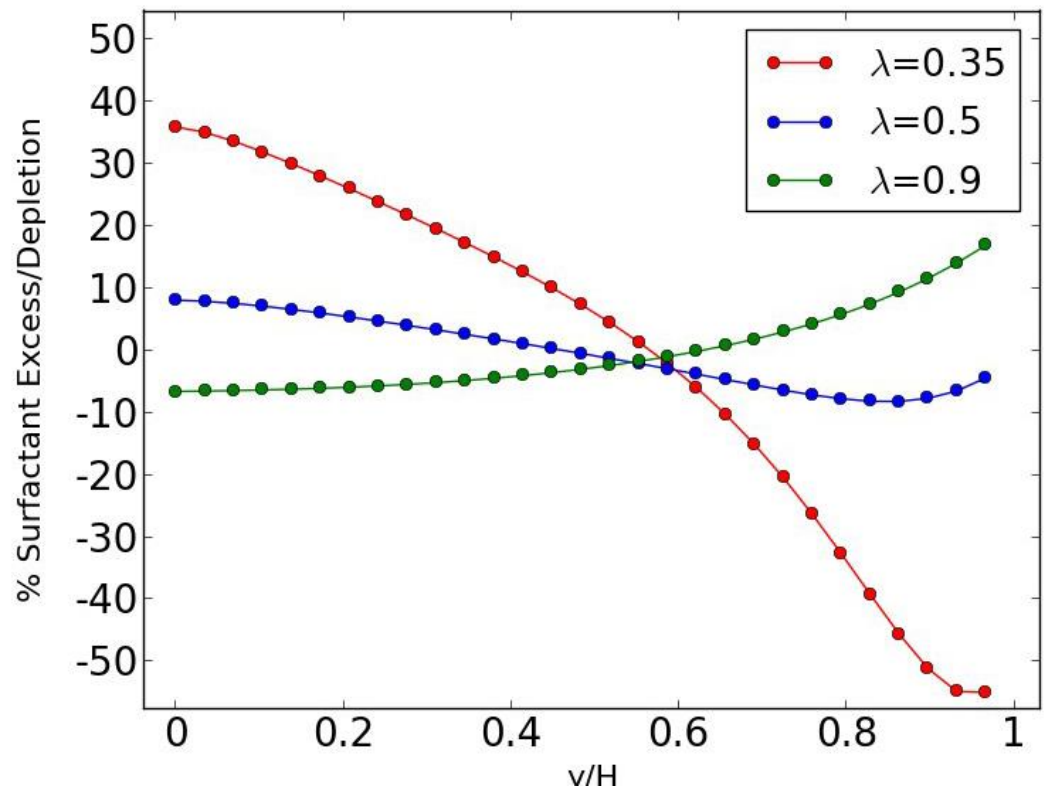

Figure S5. Deformation by wet sintering. Percent surfactant excess/depletion as a function of scaled distance from the substrate in the vertical direction for different values of $\bar{\lambda}$. $\mathrm{k}=4 ; \overline{\mathrm{A}}=1 ; \mathrm{P}_{\mathrm{eP}}=50 ; \mathrm{P}_{\mathrm{eS}}=0.5 ; \mathrm{t}=1.25$. 


\section{Derivation of the transport equations by means of a variational principle}

We provide below a derivation of the equations for the transport of latex beads and surfactant molecules, based on a least dissipation variational approach. The approach is based on a Rayleighian function $\mathcal{R}=\dot{\mathcal{G}}+\mathcal{D} / 2$ sum of the Gibbs free-energy variation rate $\dot{\mathcal{G}}$ and one half of the dissipation rate $\mathcal{D} / 2$. The minimization of $\mathcal{R}$ with respect to each thermodynamic variable variation rate provides a set of dynamic equations, which can be interpreted as the kinetic linear response to the thermodynamic forces acting in the system (Onsager relaxation principle). Our derivation follows closely Doi [M. Doi, Onsager's Variational Principle in Soft Matter, Journal of Physics : Condensed Matter, 2011, 23, 284118 and Soft Matter Physics, Oxford University Press, 2013].

The free energy variation rate $\dot{\mathcal{G}}$ originates from a gradient of surfactant chemical potential and a gradient of osmotic pressure for the concentrated suspension (chemical potential of the beads). In expressing the first contribution, one must take care of the Langmuir equilibrium between surfactant adsorbed on the beads, and in solution. This equilibrium is assumed to hold everywhere and at all times.

Langmuir equilibrium and latex beads free-energy

Taking the latex solution as a suspension of identical spheres with radius $R_{0}$ and number concentration $C_{p}$. The bead volume fraction follows from simple geometrical considerations

$$
\phi=\frac{4 \pi R_{0}^{3}}{3} C_{p}
$$

The surface to volume ratio of the suspension is $3 / R_{0}$. In what follows, it turns out to be convenient to express the surfactant concentrations in terms of volume fraction $\phi$. We therefore introduce $(1-\phi) C_{s}$, average number density of free surfactant in solution and $\phi C_{a}$, equivalent fraction of surfactant adsorbed onto the surface. The total number of surfactant molecules in a volume reads

$$
\int \mathrm{d} \mathbf{r}\left[(1-\phi) C_{s}+\phi C_{a}\right]
$$

The Langmuir equilibrium rules the exchange of surfactant molecules at concentration $C_{s}$ in the solvent with single occupancy adsorption sites at the surface, and is characterized by an adsorption energy $-\varepsilon$ and a maximal coverage density $\phi C_{m}$. In other words, each latex bead carries at most $\frac{4 \pi R_{0}^{3}}{3} C_{m}$ on its surface $\left(R_{0} C_{m} / 3\right.$ molecules per unit surface). Within the previous assumptions and notations, the chemical potential of surfactant molecules adsorbed at the surface reads $\mu_{a}=k T C_{a} /\left(C_{m}-C_{a}\right)-\varepsilon$ and must match the chemical potential in solution $\mu_{s}$. If in addition $\mu_{s}$ follows the Raoult law, the Langmuir equilibrium reduces to a standard relation between surface and volume concentrations

$$
\frac{C_{a}}{C_{m}}=\frac{C_{s}}{C_{s}+A},
$$

$A$ being the volume concentration corresponding to a half-covered bead surface. In deriving the transport equations of both surfactant and particle beads, one must express the total Gibbs free-energy variation rate. The surfactant contribution to $\dot{G}$ can be easily expressed in terms of chemical potential

$$
\int \mathrm{d} \mathbf{r}\left[\mu_{a} \frac{\partial}{\partial t}\left(\phi C_{a}\right)+\mu_{s} \frac{\partial}{\partial t}\left((1-\phi) C_{s}\right)\right]
$$


Given that Langmuir equilibrium is assumed throughout the sample, one has $\mu_{s}=\mu_{a}$ and the expression becomes

$$
\int \mathrm{d} \mathbf{r} \mu_{s} \frac{\partial}{\partial t}\left[\phi C_{a}+(1-\phi) C_{s}\right]
$$

The second contribution to the Gibbs free-energy variation rate originates from excluded volume, dispersion and electrostatic interactions. All these are summarized into a free-energy per volume $f(\phi)$. The contribution to $\dot{G}$ simply reads

$$
\int \mathrm{d} \mathbf{r} f^{\prime}(\phi) \frac{\partial \phi}{\partial t}
$$

It is clear from eq (5) and (6) that the thermodynamic variables under considerations are $\phi$ and $C_{s}\left(C_{a}\right.$ being related to them through Langmuir equilibrium). These are the quantities for which a transport equation must be found.

\section{Dissipation for moving beads and surfactants in a solvent at rest}

The simplest model assumes that solvent particles remain, on average, at rest. Introducing the average velocity $\mathbf{v}_{s}$ and diffusion coefficient $D_{s}$ of surfactant molecules, one can express the viscous dissipation of molecules as

$$
\int \mathrm{d} \mathbf{r} \frac{k T}{D_{s}} \mathbf{v}_{s}^{2}(1-\phi) C_{s}
$$

One recognizes in the above expression the particle friction coefficient $k T / D_{s}$ (Einstein relation). The product $(1-\phi) C_{s} \mathrm{~d} \mathbf{r}$ gives the number of surfactant molecules occupying a mesoscopic volume $\mathrm{d} \mathbf{r}$, while $C_{s}$ is the local concentration of tensioactive molecules, linked to the chemical potential $\mu_{s}$.

An analogue expression accounts for the dissipation of latex beads drifting across the static medium. Denoting $\mathbf{v}_{p}$ the local average bead velocity, the dissipation term reads

$$
\int \mathrm{d} \mathbf{r} \frac{k T}{D_{c}(\phi)} \mathbf{v}_{p}^{2} \frac{3 \phi}{4 \pi R_{0}^{3}}
$$

The $\phi$ dependence of the friction $k T / D_{c}(\phi)$ expresses the influence of hydrodynamic interactions acting in concentrated beads solutions, while the dilute limit $k T / D_{c}(\phi \simeq 0)=6 \pi \eta R_{0}$ reduces to the usual Stokes friction.

\section{Rayleighian and transport equations}

We obtain for a solvent at rest the following Rayleighian

$$
\mathcal{R}=\int \mathrm{d} \mathbf{r}\left\{\mu_{s} \frac{\partial}{\partial t}\left(\phi C_{a}+(1-\phi) C_{s}\right)+f^{\prime}(\phi) \frac{\partial \phi}{\partial t}+\frac{k T}{2 D_{s}} \mathbf{v}_{s}^{2}(1-\phi) C_{s}+\frac{k T}{2 D_{c}(\phi)} \mathbf{v}_{p}^{2} \frac{3 \phi}{4 \pi R_{0}^{3}}\right\}
$$

the dynamic variables of interest being $\partial \phi / \partial t$ and $\partial C_{s} / \partial t$. They are related to drift velocities and currents by the usual flux balance relations :

$$
\frac{\partial \phi}{\partial t}+\nabla \cdot\left(\mathbf{v}_{p} \phi\right)=0
$$

for latex beads, and

$$
\frac{\partial}{\partial t}\left(\phi C_{a}+(1-\phi) C_{s}\right)+\nabla \cdot\left(\mathbf{v}_{p} \phi C_{a}+\mathbf{v}_{s}(1-\phi) C_{s}\right)=0
$$


for surfactant molecules. These two relations makes it possible to rewrite the Rayleighian in terms of bead and surfactant velocities only. Integrating by part, and assuming that no flux of surfactant or particles is allowed across the system boundaries, one obtains

$\mathcal{R}=\int \mathrm{d} \mathbf{r}\left\{\left(\phi C_{a} \mathbf{v}_{p}+(1-\phi) C_{s} \mathbf{v}_{s}\right) \cdot \nabla \mu_{s}+\Pi^{\prime}(\phi) \mathbf{v}_{p} \cdot \nabla \phi+\frac{k T}{2 D_{s}} \mathbf{v}_{s}^{2}(1-\phi) C_{s}+\frac{k T}{2 D_{c}(\phi)} \mathbf{v}_{p}^{2} \frac{3 \phi}{4 \pi R_{0}^{3}}\right\}$,

where the identity $\phi \nabla f^{\prime}(\phi)=\Pi^{\prime}(\phi) \nabla \phi$ is used to put the latex beads osmotic pressure $\Pi(\phi)=$ $\phi f^{\prime}(\phi)-f(\phi)+f(0)$ in evidence.

Setting the functional derivatives of $\mathcal{R}$ with respect to $\mathbf{v}_{p}(\mathbf{r})$ and $\mathbf{v}_{s}(\mathbf{r})$ to zero (the OnsagerRayleigh variational principle) determines both $\mathbf{v}_{p}$ and $\mathbf{v}_{s}$ in terms of gradients of $\phi$ and $\mu_{s}$.

$$
\begin{aligned}
\frac{k T}{D_{s}} C_{s}(1-\phi) \mathbf{v}_{s}+(1-\phi) C_{s} \boldsymbol{\nabla} \mu_{s} & =0 ; \\
\Pi^{\prime}(\phi) \boldsymbol{\nabla} \phi+\frac{k T}{D_{c}(\phi)} \frac{3 \phi}{4 \pi R_{0}^{3}} \mathbf{v}_{p}+\phi C_{a} \boldsymbol{\nabla} \mu_{s} & =0 .
\end{aligned}
$$

It is then straightforward to substitute for $\mathbf{v}_{p}$ and $\mathbf{v}_{s}$ in eqs $(10,11)$, resulting in the transport equations for beads and surfactant. In the following expressions, gradients are given in terms of the vertical variable $y$.

$$
\begin{gathered}
\frac{\partial \phi}{\partial t}-\frac{\partial}{\partial y}\left(\frac{4 \pi R_{0}^{3}}{3} \Pi^{\prime}(\phi) \frac{D_{c}(\phi)}{k T} \frac{\partial \phi}{\partial y}\right)-\frac{\partial}{\partial y}\left(\frac{D_{c}}{k T} \frac{4 \pi R_{0}^{3}}{3} \phi C_{a} \frac{\partial \mu_{s}}{\partial y}\right)=0 \\
\frac{\partial}{\partial t}\left(\phi C_{a}+(1-\phi) C_{s}\right)-\frac{\partial}{\partial y}\left(\frac{D_{s}}{k T}(1-\phi) C_{s} \frac{\partial \mu_{s}}{\partial y}+\frac{D_{c}}{k T} \frac{4 \pi R_{0}^{3}}{3} \phi C_{a} \frac{\partial \mu_{s}}{\partial y}+\Pi^{\prime}(\phi) \frac{D_{c}}{k T} \frac{4 \pi R_{0}^{3}}{3} C_{a} \frac{\partial \phi}{\partial y}\right)=0 .
\end{gathered}
$$

Let us describe briefly the terms appearing in the above equations. Eq (15) relates the time variation of the bead volume fraction to the gradient of osmotic pressure (middle term) and a crossed diffusio-osmotic contribution proportional to the gradient of chemical potential $\mu_{s}$ (last term). This term is present because each latex bead carries along a number of adsorbed surfactant molecules proportional to $\phi C_{a}$, and therefore feels a thermodynamic force pushing it towards the chemical potential $\mu_{s}$ descent direction (see eq 14). In the improved treatment that follows below, we show that a different and more physical diffusio-osmotic term emerges, that is usually neglected in practice. Eq (16) relates the variation of surfactant molecules concentration (first term) to the gradient of chemical potential (second term) and the advection of surfactant molecules adsorbed on latex beads (last term). The third term is a counterpart of the crossed diffusio-osmotic term discussed above.

Neglecting the diffusio-osmotic term $\phi C_{a} \nabla \mu_{s}$ in eq (14) and assuming an ideal behavior for $\mu_{s}=\mu_{s}^{(0)}+k T \ln \left(C_{s} / C_{0}\right)$ leads to a basic set of equations, that constitute the starting point of the study by Gundabala et al.

$$
\begin{gathered}
\frac{\partial \phi}{\partial t}-\frac{\partial}{\partial y}\left(D_{p}(\phi) \frac{\partial \phi}{\partial y}\right)=0 \\
\frac{\partial}{\partial t}\left(\phi C_{a}+(1-\phi) C_{s}\right)-\frac{\partial}{\partial y}\left(D_{s}(1-\phi) \frac{\partial C_{s}}{\partial y}+D_{p}(\phi) C_{a} \frac{\partial \phi}{\partial y}\right)=0 .
\end{gathered}
$$


with a particle collective diffusion coefficient defined as

$$
D_{p}(\phi)=\frac{4 \pi R_{0}^{3}}{3} \frac{\Pi^{\prime}(\phi)}{k T} D_{c}(\phi)
$$

The connection with Gundabala et al follows from the following additional notations and adimensionalisation : $C_{s 0}$ initial surfactant concentration, $C_{a} \equiv 3 \Gamma / R_{0}, 3 \Gamma_{\infty} / R_{0} \equiv C_{m}, \overline{C_{s}} \equiv C_{s} / C_{s 0}$, $\bar{\Gamma} \equiv 3 \Gamma /\left(R_{0} C_{s 0}\right)=C_{m} / C_{s 0}$.

\section{Water exchange due to latex beads displacement}

To improve upon the steady solvent assumption, we now account for the solvent incompressibility by modifying the conservation equation for beads and stating that the displacement of a bead results in the exchange with an equivalent volume of solvent moving opposite to the bead. This results in a different transport equation for the surfactants

$$
\frac{\partial}{\partial t}\left(\phi C_{a}+(1-\phi) C_{s}\right)+\nabla \cdot\left(\mathbf{v}_{p} \phi C_{a}-\mathbf{v}_{p} \phi C_{s}+\mathbf{v}_{s}(1-\phi) C_{s}\right)=0 .
$$

Reiterating the above steps leads to a modified Rayleighian

$$
\begin{aligned}
\mathcal{R}^{\prime}= & \int \mathrm{d} \mathbf{r}\left\{\left(\phi\left(C_{a}-C_{s}\right) \mathbf{v}_{p}+(1-\phi) C_{s} \mathbf{v}_{s}\right) \cdot \nabla \mu_{s}+\Pi^{\prime}(\phi) \mathbf{v}_{p} \cdot \nabla \phi\right. \\
& \left.+\frac{k T}{2 D_{s}} \mathbf{v}_{s}^{2}(1-\phi) C_{s}+\frac{k T}{2 D_{c}} \frac{3 \phi}{4 \pi R_{0}^{3}} \mathbf{v}_{p}^{2}\right\}
\end{aligned}
$$

and to the following dissipation equations

$$
\begin{aligned}
\phi \mathbf{v}_{p} & =-\frac{4 \pi R_{0}^{3}}{3} \frac{D_{c}}{k T}\left(\Pi^{\prime}(\phi) \nabla \phi+\phi\left(C_{a}-C_{s}\right) \nabla \mu_{s}\right) ; \\
C_{s} \mathbf{v}_{s} & =-\frac{D_{s}}{k T} C_{s} \boldsymbol{\nabla} \mu_{s} .
\end{aligned}
$$

A significant difference compared with the steady solvent case concerns the diffusio-osmotic term $\phi\left(C_{a}-C_{s}\right) \nabla \mu_{s}$. As expected, the gradient in chemical potential is now coupled the difference $C_{a}-C_{s}$ and the sign of the effective force is a priori undetermined. It depends on whether the amount of surfactant carried by a colloidal particle is higher or lesser than the amount of surfactant occupying an equivalent volume of solution. Neglecting again this contribution and combining with eq (20) leads to a slightly different set of equations, which account properly for a surfactant transport by a backflow around colloidal particles.

$$
\begin{gathered}
\frac{\partial \phi}{\partial t}-\frac{\partial}{\partial y}\left(\frac{4 \pi R_{0}^{3}}{3} \Pi^{\prime}(\phi) \frac{D_{c}(\phi)}{k T} \frac{\partial \phi}{\partial y}\right)=0 \\
\frac{\partial}{\partial t}\left(\phi C_{a}+(1-\phi) C_{s}\right)-\frac{\partial}{\partial y}\left(D_{s}(1-\phi) \frac{\partial C_{s}}{\partial y}+\Pi^{\prime}(\phi) \frac{D_{c}}{k T} \frac{4 \pi R_{0}^{3}}{3}\left(C_{a}-C_{s}\right) \frac{\partial \phi}{\partial y}\right)=0 .
\end{gathered}
$$

Simplifying by means of the collective diffusion constant $D_{p}$, one finally obtains :

$$
\frac{\partial \phi}{\partial t}-\frac{\partial}{\partial y}\left(D_{p}(\phi) \frac{\partial \phi}{\partial y}\right)=0
$$




$$
\frac{\partial}{\partial t}\left(\phi C_{a}+(1-\phi) C_{s}\right)-\frac{\partial}{\partial y}\left(D_{s}(1-\phi) \frac{\partial C_{s}}{\partial y}+D_{p}(\phi)\left(C_{a}-C_{s}\right) \frac{\partial \phi}{\partial y}\right)=0 .
$$

These equations are solved and compared to the results of Gundabala et al.

We note that for better consistency, diffusio-osmotic terms should be kept, and the non vanishing solvent velocity should also be accounted for in the dissipation terms. Hindered surfactant diffusion could also been introduced by means of a $\phi$ dependent coefficient $D_{s}(\phi)$. This leads to ever more complex transport equations that will be the subject of future work. 